WORKING PAPER $\cdot$ NO. 2020-17

\title{
Political Scandal: A Theory
}

Wioletta Dziuda and William G. Howell

MARCH 2020 


\title{
Political Scandal: A Theory*
}

\author{
Wioletta Dziuda ${ }^{\dagger}$ \\ University of Chicago
}

\author{
William G. Howell ${ }^{\ddagger}$ \\ University of Chicago
}

March 14, 2020

\begin{abstract}
We study a model that characterizes the conditions under which past misbehavior becomes the subject of present scandal, with consequences for both the implicated politician and the parties that work with him. In the model, both authentic and fake scandals arise endogenously within a political framework involving two parties that trade off benefits of continued collaboration with a suspect politician against the possibility of reputational fallout. Rising polarization between the two parties, we show, increases the likelihood of scandal while decreasing its informational value. Scandals that are triggered by only the opposing party, we also find, are reputationally damaging to both parties and, in some instances, reputationally enhancing to the politician. The model also reveals that jurisdictions with lots of scandals are not necessarily beset by more misbehavior. Under well-defined conditions, in fact, scandals can be a sign of political piety.
\end{abstract}

${ }^{*}$ For helpful feedback on earlier versions of this paper, we thank Avidit Acharya, Scott Ashworth, David Austen-Smith, Ethan Bueno de Mesquita, Dan Bernhardt, Peter Buisseret, Gabriele Gratton, Giovanna Invernizzi, Frances Rosenbluth, Brandon Rottinghaus, Stephane Wolton and the participants of PIEP conference. Standard disclaimers apply.

${ }^{\dagger}$ Assistant Professor; Harris School of Public Policy; 1307 E University Ave; Chicago, IL 60637; wdziuda@uchicago.edu

${ }^{\ddagger}$ Sydney Stein Professor of American Politics; Harris School of Public Policy; 1307 E University Ave; Chicago, IL 60637; whowell@uchicago.edu 


\section{Introduction}

American politics is awash in scandal. The most renowned of them-Teapot Dome, Watergate, Iran-Contra, Monica Lewinsky, Russian collusion-consumed presidents. But outside of the White House, plenty more transgressions, ill-gotten gains, moral lapses, lies, and crimes have derailed the political careers of politicians. As Brandon Rottinghaus $(2015,161)$ observes, "by their nature, scandals are like prairie fires - easy to flare, difficult to control, and hard to stop once started." Indeed, outside of wars and economic downturns, scandals may be the most disruptive and damaging force in American politics.

As a pervasive and enduring fact of political life, scandals have become the subject of serious empirical scrutiny (for summaries, see Dewberry 2015, 4-12; Rottinghaus 2015, 3-7; Invernizzi 2016). Scholars also have begun to build theory that evaluates the strategic behavior of politicians amidst political scandal (Basinger and Rottinghaus 2012; Dewan and Myatt 2012; Gratton, Holden and Kolotilin 2018). None of the existing scholarship, however, identifies specific conditions under which past misbehavior, through public revelation, translates into present political scandal - a subject that is of intrinsic interest, but that also vexes the inferences we can draw from observational studies of scandals. From both a theoretical and empirical standpoint, the political incentives that undergird the production of scandal remain opaque. As Charles Cameron $(2002,655)$ laments, "The politics of scandal has not received the degree of serious scholarly attention it probably deserves. [If] scandal seeking and scandal mongering are normal political tactics... then political scientists need to learn their logic." Or as Giovanna Invernizzi (2016, 18) notes, "we still lack a proper theoretical characterization which puts scandals in the broad context of political structures and strategic behavior of the actors involved."

At its heart, scandal is the public revelation of previously concealed misconduct (Dewberry 2015, 4-6; Thompson 2000, 18-19); or as Theodore Lowi (1988, vii) puts it, "scandal is corruption revealed." Public accusations about past misdeeds, however, need not be universally endorsed. Parties may misrepresent the information that they receive about a politician, either through suppression or fabrication. Consequentially, politics regularly features "partisan scandals," that is, accusations by one party that are vehemently denied by 
another. The politics of scandal, moreover, regularly features efforts to ascertain the veracity of accusations leveled. In addition to specifying processes by which claims of misconduct are asserted, therefore, we need theory that clarifies when "authentic" and "fake" scandals are likely to arise, and the political consequences for the implicated politicians and their associates.

To make headway on the problem, we distill the essential strategic considerations of scandal production. In the model, scandals are generated endogenously within a political framework involving two political parties - one aligned with a politician, the other opposedthat trade off benefits (in case of the aligned party) or costs (in case of the opposing party) of continued collaboration with the politician against the reputational consequences of scandal. With some probability, parties learn that the politician engaged in misconduct. The parties then must decide whether to act on this information and publicly accuse the politician of misconduct, recognizing that doing so will affect the politician's political survival and the parties' reputations for honesty. Parties also may engage in "fake news" tactics by leveling accusations even when they received no substantiating information. Any accusation of misconduct leads to a scandal. The voter, therefore, must decide when scandals reflect actual misconduct, when they are born of deceit, and when, absent any scandal at all, a politician nonetheless warrants removal from office. The implicated politician is voted out of office with probability equal to the voter's belief that he misbehaved; and, with known probability, is replaced by a new politician with a different party affiliation.

The model unearths three main findings that clarify when different kinds of scandals are likely to arise and their consequences both for the careers of politicians and the reputations of parties. First, the model reveals how polarization between parties breeds dishonesty in scandal production: the greater the disagreement between the two parties, the more likely the aligned party will suppress information about the politician's misconduct in order to keep him in power, and that the opposing party will falsely accuse the politician in order to hasten his removal. Polarization, as such, degrades public discourse, as one party accuses and the other denies, leaving voters with little ability to learn where the truth lies.

Second, we find that increases in actual misbehavior may coincide with either increases or decreases in the production of scandals - a finding with immediate implications for empirical 
literatures that interpret scandals as proxies for underlying rates of corruption. Scandals do not increase monotonically in actual misbehavior as, we show, parties are especially likely to falsely accuse a politician when base rates of misbehavior assume intermediate values.

Third, and finally, the model clarifies why it is so difficult to generalize about the reputational consequences of scandals. Scandals tend to lower parties' reputations, albeit differentially depending upon circumstances that we characterize. In all cases, however, the political fortunes of the implicated politician and his party diverge. For example, by defending a politician who stands accused by the opposing party and who is looked upon rather dimly by the voter, we show, the aligned party absorbs a scandal's political fallout - a finding that illuminates one rational for why the approval ratings of Bill Clinton and Donald Trump remained steady through much of their scandal-ridden presidencies, while the parties that defended them suffered electorally in Congress.

All told, the model makes two general contributions: one substantive, the other theoretical. Substantively, we show how a wide variety of seemingly disparate facts about scandals - whether one or both parties recognize their existence, ${ }^{1}$ the fact that equivalent behaviors can evoke very different reactions from the same party, ${ }^{2}$ and the varying reputational consequences of scandals for parties and politicians ${ }^{3}$ - can arise in a simple framework with rational voters and parties. Theoretically, our model of communication with reputational concerns by two experts with opposite preferences is, to our knowledge, novel.

We proceed as follows. After summarizing the relevant literatures on scandal and providing a motivating example, we introduce the model. We then characterize the conditions under which parties will attempt to deceive the voter and the implications of their behavior for the incidence of scandal. Subsequent sections characterize the reputational and career effects of different types of scandals and the inferential errors that voters make about them.

\footnotetext{
${ }^{1}$ Compare, for instance, Republicans' steadfast unwillingness to recognize Donald Trump's sexual improprieties and the bipartisan outrage directed at Senator Al Franken's (Yamiche Alcindor and Nicholas Fandos, "A Democratic Chorus Rises in the Senate: 'Franken Should Resign.' " New York Times, December 6, 2017.

${ }^{2}$ Compare Republicans' reaction to Representative Steve King's inflammatory remarks and virtual silence in the aftermath of Trump's (Sheryl Gay Stolberg, "As Republicans Rush to Condemn Steve King, Some Ask: Why Not Trump?" New York Times, January 16, 2019).

${ }^{3}$ As an example, compare scandal-ridden Chicago Alderman Edward Burke's 2019 reelection with the resounding mayoral defeat of Toni Preckwinkle, a Democratic party stalwart who supported Burke (Sanjana Karanth, "Chicago Alderman Ed Burke Win Re-election Despite Facing Criminal Charges," Huffington Post, February 27, 2019.)
} 
The final section discusses our findings and concludes. The Supplementary Material collects all proofs and examines the results of a model that endogenizes misbehavior.

\section{Literature Review}

Over the last two decades, a growing number of political scientists have sought to clarify the relevance of political scandal for contemporary American politics. Much of the resulting empirical scholarship focuses on the consequences of scandal, whether for its perpetrators, those associated with them, or the larger polity. In addition to negatively affecting a politician's public approval ratings (Simon and Ostrom 1989; Zaller 1998; Andolina and Wilcox 2000, Renshon 2002; Woessner 2005; Green, Zelizer, and Kiriby 2018), scandals have been shown to affect legislative voting patterns (Meinke and Anderson 2001), the strength of party identification (Chaffee and Becker 1975; Dunlap and Wisniewski 1978; Robinson 1974); the nation's policy agenda and inter-branch relations (Rottinghaus 2015), media coverage of politics (Sabato, Stencel, and Lichter 2001; Puglisi and Snyder 2011; Entman 2012), public trust in government and its assessments of political institutions (Lipset and Schneider 1983; Miller 1999; Bowler and Karp 2004; Green, Zelizer, and Kirby 2018), voter assessments of individual candidates (Lipset and Schneider 1983; Carlson, Ganiel, and Hyde 2000; Funk 1996; Banerjee et al 2014; Green, Zelizer, and Kirby 2018), and the outcome of subsequent elections (Welch and Hibbing 1997; Klasnja 2017; Peters and Welch 1980; Pereira and Waterbury 2018; Jacobson and Dimock 1994; Hirano and Snyder 2018; Chong et al 2015).

When are these various disruptions most likely to occur? For answers, scholars have scrutinized the conditions under which past misbehavior turns to present scandal. Some, particularly journalists, emphasize the importance of individual politicians' characters and personal relations (see, for example, Woodward and Bernstein 1974; Toobin 2000; Coen and Chase 2012; Harding 2017; Bongino and McAllister 2018). Political forces, though, also play a part, and political scientists have documented numerous predictors of scandal frequency and duration, including the incidence of divided government (Sowers and Nelson 2016), poverty and political corruption (Nice 1983), the number of other topics vying for news coverage (Nyhan 2015), low approval ratings (Nyhan 2017), and a variety of cultural, 
historical, and bureaucratic forces (Meier and Holbrook 1992).

Diverse data support these empirical findings, including content analyses of media coverage (Rottinghaus 2015; Nyhan 2015, 2017), expert surveys about corruption perception (Mishler and Rose 2001; Anderson and Tverdova 2003; Boyland and Long 2003), and judicial convictions (Hirano and Snyder 2018). The validity and reliability of such measures are matters of ongoing dispute, as scholars have raised concerns about the changing norms of scandal coverage over time (Adut 2005, 2008), the correlations between convictions for and media perceptions of political corruption (Boylan and Long 2003), and competing definitions of what constitutes a scandal (see Rottinghaus 2015, 18-20; Thompson 2000, 11-30).

For each of their individual strengths and weaknesses, however, all measures within the existing empirical literature document publicly observed scandals. In one way or another, each is based upon the judgments of the media, prosecutors, or experts about the incidence of specific public scandals or impressions of their general occurrence. And as purely descriptive exercises, this is fine and well. But to the extent that we are interested in using these data to make inferences about underlying transgressions, this reliance on publicly observed scandals is highly problematic. Scandals, after all, do not represent a random draw of political misbehavior. As we have learned from those rare instances when a randomized audit has been conducted (see, for example, Ferraz and Finan 2011), patterns of corruption do not map neatly onto patterns of scandal.

To make sense of these politics, it will not do to simply correlate measures of observed scandals against descriptors of the political environment. Politicians who are prone to misbehavior and those who would report their misdeeds, after all, can be expected to strategically adapt to changes in this environment. As Nyhan $(2017,33)$ notes, "the media scandals that so often dominate the headlines are not exogenous but instead the result of a fundamentally political process. We cannot understand when and why [politicians] suffer from scandals without considering the role of strategic behavior and the context in which events take place." 4

To clarify this "fundamentally political process," we need theory that identifies specific

\footnotetext{
${ }^{4}$ This point is further underscored by a nascent literature that investigates how observable signs of corruption relate to its actual incidence; see, for example, Chassang and Padro i Miguel (forthcoming).
} 
conditions under which misdeeds are more or less likely to be publicly revealed, and the propensity of would-be perpetrators, a priori, to adjust accordingly. Just now, though, we know very little about the political logic that translates misbehavior (however defined) into scandal (however observed). Though a number of scholars have begun to build theories that feature scandal (Basinger and Rottinghaus 2012; Dewan and Myatt 2012; Gratton, Holden and Kolotilin 2018), none answers a question of rudimentary importance in the politics of scandal: when, and with what consequence, is misbehavior likely to be exposed? On this front, Besley and Prat (2006) analyze the role of politically influenced media in revealing politicians' misbehavior. They do not allow for fake scandals nor do they consider competition between media with very different dispositions toward the politician. Like us, Besley and Prat find that the relationship between misconduct and scandals is nonmonotone, but their finding comes solely from a strategic response by the politician, while in our model, the nonmonotonicity arises through the endogenous actions of parties levelling accusations about exogenously determined misbehavior.

\section{Two Motivating Examples}

Not all scandals are of a piece. Nor are their politics. Depending upon the relationships of the actors involved, the nature of the informational environment, and the type of accusations under scrutiny, very different political dynamics may follow. When building theory, therefore, it is important to be clear about the specific type of scandal one intends to characterize.

The Trump presidency has furnished ample scandals that have characteristics we want to capture. Let's consider just one that, while not the most famous or consequential, does a particularly nice job of illustrating the central features of our model. The key events occurred during a bipartisan meeting Donald Trump held with congressional delegates on January 11, 2018. The meeting was noteworthy not for any policy disagreements it settled, but instead for something the president may have said behind closed doors. When leaving the meeting, congressional Democrats claimed that Trump disparaged immigrants from "shithole countries." 5 Immediately thereafter a public row ensued, as advocates for immigrants' rights,

\footnotetext{
${ }^{5}$ Josh Dawsey, "Trump derides protections for immigrants from 'shithole' countries." Washington Post,
} 
the diplomatic teams from some of the targeted countries, and leaders of the Democratic Party all took the president to task for his uncouth - and, said some, racist-language.

Republicans who attended the meeting, however, refused to corroborate the charges of their Democratic colleagues. Secretary of Homeland Security Kirstjen Nielsen testified under oath that, "I did not hear that word [shithole] used... The president used tough language in general, as did other congressmen in the room." Senators Tom Cotton (R-AR) and David Perdue (R-GA), who also were in attendance, claimed that Trump referred to "shithouse" and not "shithole" countries. ${ }^{6}$ In the days that followed, the public struggled to make sense of these competing accounts and what they revealed about both the president and those who subsequently reported on his behavior.

We find here the main elements of the types of scandals that we intend to model. Everyone who attended the January, 2018 White House meeting heard the president, but whereas his opponents insisted that he used vulgar language to disparage immigrants, his allies equivocated. Without any independent way to verify their claims, meanwhile, voters were left to wonder whether the president had in fact misbehaved, and whether it was the Democrats or Republicans who subsequently lied about it. Discussions about simple matters of fact, as such, were rather promptly imbued with additional concerns about the political motivations of all involved.

Our model also captures situations in which certain facts concerning alleged misbehavior are public knowledge, but voters remain uncertain about whether the parties posses other useful information. For example, when Senator Al Franken (D-MN) was accused of sexual misconduct in 2017, the photograph documenting his questionable behavior was public and not disputed, and most likely affected voters' perception of his guilt. Quite reasonably, though, voters could expect that senators possessed other relevant information about Franken's past interactions with women. Voters therefore had reason to make inferences about Franken's misbehavior, and whether it warranted his removal from office, on the basis of what the Democratic and Republican parties had to say about them.

\footnotetext{
January 12, 2018.

${ }^{6}$ Andrew Prokop, "The 'shithouse defense,' explained: how Trump's allies are trying to dig him out of his 'shithole.' " Vox, January 16, 2018.
} 


\section{A Model}

We study a political environment that includes four actors: an aligned party ("it"), an opposing party (also "it"), a politician ("he"), and a voter ("she"). With probability $\pi$, the politician misbehaved - that is, committed an act that, if revealed, would constitute a scandal. Parameter $\pi$ is therefore interpreted as the underlying incidence or prior perception of misbehavior. ${ }^{7}$ Let $m \in\{0,1\}$ be a random variable denoting whether or not the politician misbehaved. If $m=1$, then with probability $p$, both parties learn about the misbehavior, and with the remaining probability, neither party learns about misbehavior. The parameter $p$, which reflects the discoverability of misbehavior, may assume different values depending on the degree of easiness with which politician's misbehavior can be observed by the parties. Let $v \in\{0,1\}$ be a random variable denoting whether or not the parties have information about the politician's misbehavior.

Each party $i \in\{$ align,opp $\}$ chooses an action $a_{i} \in\{0,1\}$ independent of the information it has about the politician's misconduct. Action $a_{i}=1$ is interpreted as unleashing a scandal, and action $a_{i}=0$ is interpreted as remaining silent. Hence, the choice set of both parties is not constrained by the information they receive. Each party may choose to honestly report misbehavior when it learns about it $\left(a_{i}=1\right.$ when $\left.v=1\right)$ or to honestly remain silent when it does not $\left(a_{i}=0\right.$ when $\left.v=0\right)$. But both parties also are free to suppress information they have learned $\left(a_{i}=0\right.$ when $\left.v=1\right)$ or to fabricate accusations in the absence of information $\left(a_{i}=1\right.$ when $\left.v=0\right)$. Such fabrication reflects instances when mere rumors about a politician's misbehavior lead to calls for his dismissal, even though the parties involved have no corroborating information about the charges involved. We refer to any accusation of misbehavior, be it based on observed information or the result of fabrication, as a scandal. $^{8}$

\footnotetext{
${ }^{7}$ As such, $\pi$ can be interpreted as the latent probability that the politician would misbehave, the strength of a rumor about the politician's misbehavior, or the chances that the politician was involved in some publicly known scandal. It can reflect all verifiable evidence that is disclosed to the voters before the game described in this section unfolds. In this main body of this paper, the politician is nonstrategic, so $\pi$ is exogenous. In the Supplementary Material, however, we solve a version of the model that endogenizes misbehavior.

${ }^{8}$ Illustrative examples of both kinds of deception abound. Throughout the Trump presidency, congressional Republicans routinely denied knowing about any corroborating evidence concerning the subjects of the president's past misdeeds, be they communications with the Russian government, financial conflicts of interest, or ethnical breaches by his family members. When assuming the role of aligned party in our
} 
The politics of scandal, Thompson (2000, 245-59) reminds us, are imbued with concerns about reputation and trust. To account for elements of these politics, we allow each party to be one of two types: honest (probability $\gamma$ ) or strategic (probability $1-\gamma$ ). If a party is honest, then it automatically and immediately reveals any information about the politician's misbehavior; and when it does not receive information about misbehavior, the honest party remains silent. The strategic party optimally chooses $a_{i} \in\{0,1\}$ to maximize its payoff.

We make two assumptions about the processes by which accusations of misbehavior are leveled and the voter's updating of beliefs. First, we assume that the voter cannot independently corroborate claims of misconduct that are leveled by the parties. The voter's ability to ascertain the veracity of charges against a politician, therefore, depends upon the parties' incentives to truthfully report the information they receive. Second, we assume that the electorate is fully Bayesian. The voter, as such, updates her views about the parties, the politician, and the incidence of misbehavior even if no scandal occurs.

To summarize, we consider a one-period game with the following timing:

1. Nature chooses the random variable $m$, which denotes the incidence of misbehavior.

2. If the politician misbehaved, in which case $m=1$, then with probability $p \in(0,1)$ both of the parties learn its value, and $v=1$. With the remaining probability, or if $m=0$, the parties learn nothing, and $v=0$.

3. Both parties simultaneously and independently decide whether to publicly claim that they received information about misbehavior, denoted by action $a_{i} \in\{0,1\}$.

model rather than implicated politician, Trump has done much the same for his political partners both at home and abroad. When in the fall of 2018 the Saudi Crown Prince was accused of ordering the killing of Saudi dissident and U.S. citizen Jamal Khashoggi, Trump steadfastly ignored the findings of his own Central Intelligence Agency and insisted that "we may never know" who ultimately was responsible.

Fake scandals also are endemic to modern political life. In May 1993, for instance, seven workers in the White House Travel Office were summarily fired, Republican critics falsely charged, in order to make room for the cronies of newly elected president Bill Clinton. More recently, Democrats falsely accused Trump of having removed the statue of Martin Luther King from the White House, and Trump falsely accused Barack Obama of being a Muslim born in a foreign country, ordering that the Trump Tower be wiretapped during the 2016 presidential election, and planting a spy within his campaign for the presidency. And lest you think fake scandals are a distinctly American phenomenon, consider what happened in Poland after a 2010 plane crash that killed the president and many high-ranking dignitaries: the party affiliated with the deceased president falsely claimed to have evidence that the governing party conspired with Russia to commit murder, yielding an international scandal that lacked any factual basis. 
4. The voter observes $\left(a_{a l i g n}, a_{\text {opp }}\right)$ and updates her beliefs about each party's type and the occurrence of misbehavior by the politician.

5. The politician is replaced with probability equal to the voter's beliefs that he misbehaved, and the respective benefits from collaboration are realized.

The strategic party obtains payoff from two sources. The first source is its reputation for honesty, which depends on the belief that the voter holds about its type at the end of the game. ${ }^{9}$ The value of such reputational concerns can be understood either intrinsically or instrumentally, such that parties with higher reputations are electorally advantaged. Given the action of the aligned party $a_{\text {align }}$ and the opposing party $a_{\text {opp }}$, let $\phi_{i}\left(a_{\text {align }}, a_{\text {opp }}\right)$ denote the voter's beliefs about party $i$ 's type and $\Phi\left(a_{\text {align }}, a_{\text {opp }}\right)$ denote the voter's beliefs about whether misconduct occurred.

The second source of payoff concerns each party's benefits from continued collaboration. We assume that the politician is dismissed with probability equal to voter's belief that the politician misbehaved $\Phi\left(a_{\text {align }}, a_{\text {opp }}\right)$. If the politician is not dismissed, each party is guaranteed to receive its allotted collaboration payoff at the end of the game, $x_{i}$. For the aligned party, we assume $x_{a l i g n}>0$, and for the opposing party, $x_{\text {opp }}<0$. In the analysis below, we assume symmetry between the two parties' collaborative gains and losses, $x_{\text {align }}=-x_{\text {opp }} \doteq x$. One natural interpretation, and the one we adopt in what follows, is that $x$ is a marker of political polarization. The more polarized the parties are, after all, the more they benefit from having their own member in power, and the more they suffer from an opposing politician holding power. This parameter, however, proxies many other political variables that affect the returns to collaboration, such as the importance of the position held by the politician (see Section 7.7).

If the politician is dismissed, however, the returns to each party depend on the identity of his replacement, which we capture with the parameter $c \in[-1,1]$. If $c=1$, then the new politician has the same political allegiance as the old. If $c=-1$, however, the new

\footnotetext{
${ }^{9}$ Then-Commerce Secretary Herbert Hoover recognized precisely this reputational benefit when he offered the following counsel to his beleaguered President Warren Harding about how best to handle incriminating information about offending members of his administration: "Publish it," Hoover intoned, "and at least get the credit for integrity on your side" (as quoted in Whyte 2017, 283).
} 
politician's political allegiances flip. We interpret $c$ as a measure of the aligned party's political entrenchment, with $c$ reflecting the propensity of voters to punish the party of the implicated politician in any particular race.

The strategic party's utility function is therefore

$$
\phi_{i}\left(a_{\text {align }}, a_{\text {opp }}\right)+\left(1-\Phi\left(a_{\text {align }}, a_{\text {opp }}\right)\right) x_{i}+\Phi\left(a_{\text {align }}, a_{\text {opp }}\right) x_{i} c
$$

where the first element defines party $i^{\prime} s$ reputation for honesty, the second element represents its return from collaborating with the current politician, $x_{i}$, weighted by the probability that the politician is not fired, and the third element represents its expected return from collaborating with the politician's replacement, $x_{i} c$, weighted by the probability that the politician is fired. ${ }^{10}$

Thus stipulated, the most natural interpretation of the model, and the one we carry throughout the paper, treats the politician as the current president (or some other powerful leader), the aligned party as the president's party, and the opposing party as its opposition. By virtue of working with the president, both parties are privy to information about his misbehavior that is hidden from voters' view. This information is often non-verifiable, allowing the parties to say what they will about the president. On the basis of what the parties report publicly, voters update their views about all of the actors involved, with potential consequences for the politician's electoral viability and the parties' reputations for honesty.

Of course, though, we need not interpret these designations literally. Depending on how it is construed, the media, which is notably absent from the model, could be assigned to the roles of either the voter or the two parties. Fact-finding journalists, for instance, must decide whether to publish accusations of misbehavior and what exactly to say about them. To the extent that their actions affect the politician's electoral fortunes, such journalists might stand in for the voter in this model. Alternatively, one might think of partisan media

\footnotetext{
${ }^{10}$ We envision a setting wherein a politician who is removed from office does not participate in the subsequent election, the outcome of which depends entirely on the party's entrenchment. Alternatively, one might study a situation in which such a politician runs in the election, but his success depends on party entrenchment as well as his perceived guilt. In this case, the utility function would take different form than the one above. With appropriate parametrization, however, the equilibrium conditions would be virtually identical to the ones we obtain. Details available from the authors upon request.
} 
outlets with distinct relationships with politicians (e.g., as Gentzkow, Glaeser, Goldin 2004 argue was the case in the 19th century US). To the extent that they are privy to information about the politician's misbehavior, have the option of revealing it, and benefit reputationally from appearing honest, dueling networks might stand in for parties (see also Besley and Prat 2004). And, of course, still other designations might apply. The politician might be a CEO, the aligned and opposing parties might represent competing interest groups, and the voter might be a regulatory agency. Alternatively, the politician might be an operative within an international organization, the aligned and opposing parties might be opposing member states, and the voter might be a judicial body. To satisfy the scope conditions of the model, all that matters is that distinct entities that interact with a politician learn damaging, but nonverifiable, information about him and then have an opportunity to level accusations against him before an individual or body that has the power to administer punishment.

\section{Analysis}

The equilibrium concept we apply here is that of Perfect Bayesian Equilibrium (henceforth equilibrium). In service of empirical relevance, we focus the analysis on equilibria in which the opposing party never suppresses information about misbehavior, and the aligned party never fabricates it. ${ }^{11}$ Hence, any equilibrium considered in this paper is fully characterized by the conditional probability that the strategic type of the aligned party who learns about misbehavior suppresses it, denoted by $s \in[0,1]$, and by the conditional probability that the strategic type of the opposing party who does not learn about misbehavior fabricates accusation, denoted by $f \in[0,1]$.

Our first proposition stipulates the existence of an equilibrium, and shows that multiplicity of equilibria is limited.

\footnotetext{
${ }^{11}$ Our focus rules out only one equilibrium, and in this equilibrium, the voter cannot draw any inference from scandals (see Supplementary Material). One may wonder why are there no equilibria in which the aligned party fabricates information to improve its reputation. If that were the case, then the aligned party would have a strict incentive to reveal all misbehavior, which would mean that the strategic type of the aligned party would accuse more frequently than the honest type. As a result, accusations levied by the aligned party would lead to reputation loss, so the aligned party would choose not to fabricate.
} 
Proposition 1 A fully honest equilibrium with $s=f=0$ exists if and only if $x(1-c) \leq$ $2 \frac{\gamma(1-p \pi)}{1-\pi}$. An equilibrium in which parties are not fully honest exists if and only if $x(1-c)>$ $\frac{\gamma(1-p \pi)}{1-\pi}$. There exist no other equilibria. ${ }^{12}$

In the equilibria identified in Proposition 1 scandals arise either because both parties claim to have received information about misbehavior, yielding what we will call bipartisan scandals, or because only the opposition does so, yielding what we will call partisan scandals. ${ }^{13}$ We define scandal incidence to be the probability of the voter observing a scandal.

In subsequent sections, we first analyze how fabrication and suppression, the resulting incidence of scandals, and their informational value for the voter vary with the parameters of the model: stakes of an election, political entrenchment, discoverability of evidence, and prior expectations of misbehavior. In the section that follows, we analyze the consequences of scandals for the reputation of the politician and the parties. In establishing these results, whenever two equilibria coexist, we select the one that support some level of dishonesty. This equilibrium selection criterion, however, does not affect the qualitative findings that follow.

\subsection{Stakes of an election}

The statement and proof of Proposition 1 reveals that parties' incentives to suppress information or fabricate accusations depend on the difference between the payoffs from collaboration with the current politician, $x_{i}$, and the expected payoffs from collaboration with his replacement, $c x_{i}$. In subsequent propositions, therefore, we focus on their joint product, $x_{i}(1-c)$, which can be understood as the stakes of an election.

Proposition 2 summarizes the key comparative statics with respect to the stakes of an election.

Proposition 2 As stakes of an election $x(1-c)$ increase,

\footnotetext{
${ }^{12}$ For more information on the equilibrium values of $f$ and $s$, see Lemma 1 in the Appendix.

${ }^{13}$ If the aligned party was more likely to observe misbehavior, some scandals would originate with this party. Realistically, though, the opposition party would follow up with accusations of their own, so for voters such scandals may be indistinguishable from bipartisan scandals. For that reason, we expect our results would hold in a more complex model in which parties learn differentially about misbehavior but are allowed to level accusations sequentially in an order that is unobserved by the voter.
} 
1. $f$ and $s$ increase;

2. incidence of bipartisan scandals decreases;

3. incidence of all scandals increases;

4. probability that the voter makes the wrong decision (keeping a misbehaving politician or dismissing a well-behaved one) increases.

The proposition's first result is straightforward. As polarization $x$ increases,${ }^{14}$ the opposing party suffers greater collaborative losses from the sitting politician, whereas the aligned party collects greater collaborative benefits. Consequently, the former is more inclined to claim to have received information about the politician's misbehavior in order to force him out, and the latter is more inclined to suppress information to protect him. Similar incentives arise if political entrenchment of the aligned party $c$ decreases, as the aligned party is more likely to suffer and the opposing party is more likely to benefit from the sitting politician's replacement.

The proof of the first result reveals an interesting dynamic. As the stakes of election increase, each party deceives more not only because they have higher stakes in keeping or removing the politician from power, but also because the other party deceives more. Consider, for example, the aligned party. If the voter expects the opposing party to fabricate accusations due to high stakes of election, she perceives partisan scandals as likely caused by the opposing party's fabrication, and hence does not update heavily about the honesty of the aligned party. That in turn creates incentives for the aligned party to generate partisan scandals by suppressing information. In other words, an increase in stakes of an election causes a disproportionately large increase in dishonesty in scandal production, as the dishonesty of one party encourages the dishonesty of the other.

The production of scandals partially follows from the two parties' propensities to deceive, albeit not symmetrically. For a scandal to be triggered, only one party needs to allege

\footnotetext{
${ }^{14}$ Throughout, when we say "increase" or "decrease," we mean "weakly increase" or "weakly decrease" as for some parameters we have corner solutions in which the equilibrium behavior does not change further with the parameters. However, there always exist parameters for which these statements could be read "strictly increase" or "strictly decrease."
} 
misbehavior. Moreover, every time that the opposing party learns about misbehavior, regardless of whether it is honest or strategic, it will reveal the information to the voter. Hence, the aligned party's propensity to suppress information is irrelevant for the overall level of scandals, and it is the opposing party's propensity to fabricate scandals that drives scandal production. This, together with part 1, explains the second and third result of Proposition 2: the incidence of bipartisan scandals decreases in the stakes of an election due to the aligned party's higher incentive to suppress information, but the overall volume of scandals increases due to the opposing party's incentive to fabricate accusations. In this way, heightened polarization and lower party entrenchment do not merely augment scandal production. They also lend credence to charges of "fake news."

That the probability the voter commits either a Type I or Type II error is increasing in stakes of an election flows intuitively from part 1 . When the aligned party frequently suppresses information and the opposing party frequently fabricates accusations, the scandals that arise are less informative, which increases the chances that the voter will either conclude that the politician did not misbehave, when in fact he did; or that the politician did misbehave, when in fact he did not.

Overall, Proposition 2 speaks to negative consequences of polarization and party competition. An increase in ideological distance between the parties and the prospect of electoral turnover leads to a breakdown in public discourse, as one parties falsely accuses, the other denies misconduct by suspect politicians, and voters struggle to discern the truth. ${ }^{15}$

\subsection{Discoverability of misbehavior}

The probability that misbehavior is detected, $p$, surely varies according to the actions and politicians under scrutiny. Whereas the politicians present at the January 11th meeting with President Trump are all but certain to have heard what the president said, parties are less likely to have useful information on Trump's intentions behind withholding military aid to Ukraine in the summer of 2019 or Hillary Clinton's intentions behind using a private server

\footnotetext{
${ }^{15}$ Person, Tabellini and Trebbi (2003) and Eggers (2014) show that polarized voters tend not to punish misbehaving politicians. Our results imply that polarized party may result in voters having a harder time learning about misbehavior.
} 
while serving as Secretary of State under Barack Obama. Proposition 3 therefore summarizes the key comparative statics with respect to $p$.

Proposition 3 As p increases,

1. $f$ increases; s also increases, unless $f=1$, in which case s decreases: ${ }^{16}$

2. incidence of bipartisan scandals increases;

3. incidence of all scandals increases;

4. probability that the voter makes the wrong decision decreases.

Competing forces undergird these comparative statics. As $p$ increases, parties are more likely to have received information about the politician's misbehavior, and hence the voter expects a scandal. Consequentially, both parties have incentives to produce one, making suppression less likely and fabrication more likely, which pushes the dishonesty of the parties in opposite directions. As we observed in the previous section, however, forces within the model push dishonest behavior in the same direction. If the voter expects that the opposing party is likely to fabricate accusations, then should the voter observe a partisan scandal, she will interpret this as a sign of dishonesty of the opposing party and will not update much about the honesty of the aligned party. This in turn increases the aligned party incentives to suppress information. A priori, it is not obvious which effect should dominate, and Proposition 3 reveals that it is the latter.

The relationship between the likelihood that misbehavior will be discovered and the remaining variables is also not obvious, a priori. A fabrication increases, we know, the incidence of partisan scandals increases. The volume of bipartisan scandals, however, is driven by two competing forces. On the one hand, the aligned party is more likely to have information to trigger the scandal, but on the other hand, the aligned party conceals its information more frequently. Proposition 3 reveals that the former effect dominates, and the voter is more likely to see a bipartisan scandal.

Similarly, as $p$ increases, the higher dishonesty of both parties reduces the informational value of scandals, while the higher incidence of bipartisan scandals increases it. Proposition

\footnotetext{
${ }^{16}$ From Lemma 1, $f=1$ happens only for large $z$ and $\pi p>\frac{1}{2}$.
} 
3 says that the latter effect dominates, and the voter learns more from the scandals that emerge.

\subsection{Prior expectations of misbehavior}

Recall that parameter $\pi$ reflects the voter's prior belief about the likelihood that the politician misbehaved. This may reflect the overall rate of misbehavior among the political class, the voter's evaluation of the politician's character, or the strength of circulating rumors about his misbehavior. As this variable increases, it stands to reason, the voter should expect to see more scandals; and concerned about their reputations, parties ought to deliver. Naively, then, one would expect that the aligned party suppresses less and the opposing party fabricates more as $\pi$ assumes larger values. As Proposition 4 reveals, however, this intuition is incomplete.

Proposition 4 As $\pi$ increases,

1. $s$ decreases, and $f$ is single-peaked in $\pi$;

2. the incidence of bipartisan scandal increases;

3. there exists an intermediate range of $x(1-c)$ for which the overall incidence of scandal decreases.

Part 1 of Proposition 4 states that for small $\pi$, marginal increases in the voter's suspicions of misbehavior cause parties to produce more scandals: the aligned party by suppressing less and the opposing party by fabricating more. But when the voter believes that misbehavior is quite likely, the aligned party suppresses information so infrequently that the voter must interpret a partisan scandal as fabricated by the opposition. This in turn decreases the opposition party's incentive to fabricate. Hence, an increase in the incidence of misbehavior may result in fewer fake accusations. ${ }^{17}$

Proposition 4 has interesting implications for the incidence of scandals. Since the aligned party suppresses less as the incidence of misbehavior increases, the volume of bipartisan

\footnotetext{
${ }^{17}$ When $\pi$ increases, there is an additional effect. The voter perceives the politician as corrupt, and hence she is inclined to vote him out of power even in the absence of a scandal, which encourages both parties to behave more honestly.
} 
scandals increases. However, just as fabrication changes non-monotonically in changes in the underlying rates of misconduct, so does the overall incidence of scandal. In particular, if the stakes of election are intermediate, increasing the rate of misconduct may lead to a decrease in the overall volume of scandals. ${ }^{18}$

\subsection{Political Consequences of Scandal}

We turn now to identifying the political consequences of scandals. It will not do to simply estimate the average political consequences of scandals, as much of the empirical literature does. We also must scrutinize their differential effects on the reputations of various political actors. As we show in this section, scandals can have a wide range of effects on both the parties that instigate them and the politicians who stand at their center. Depending on parameters and the type of scandal, parties or the politician may suffer reputationally, they may benefit, or they may be altogether unaffected.

Let's begin with the political consequences of bipartisan scandals. Recall that $\phi_{i}\left(a_{\text {align }}, a_{\text {opp }}\right)$ denotes the voter's beliefs about party $i$ 's type and $\Phi\left(a_{\text {align }}, a_{\text {opp }}\right)$ denotes the voter's beliefs about whether misconduct occurred. After both parties allege misbehavior, the voter updates her beliefs as follows:

Proposition 5 In equilibrium,

$$
\begin{gathered}
\phi_{\text {opp }}(1,1)=\gamma \leq \phi_{\text {align }}(1,1) ; \\
\Phi(1,1)=1,
\end{gathered}
$$

where the inequality is strict if $s>0$.

The aligned party only casts accusations after having learned about misbehavior. Having observed a bipartisan scandal, therefore, the voter knows with certainty that the politician

\footnotetext{
${ }^{18}$ The omitted impact of $\pi$ on the accuracy of the voter's decision is rather obviously nonmonotonic. Even without strategic considerations, the voter is more likely to make a mistake when the incidence of misbehavior $\pi$ assumes an intermediate value. When $\pi$ approximates 1 or 0 , after all, the voter proceeds with justified confidence that the politician either did or did not misbehave. Strategic effects do not undo this general pattern, but may lead to further nonmonotonicities.
} 
misbehaved, and hence $\Phi(1,1)=1 .^{19}$ Because the strategic and honest types of the opposing party pool in this instance, however, the voter doesn't learn anything new about the opposing party's type, and hence $\phi_{\text {opp }}(1,1)=\gamma$, where $\gamma$, you will recall, is the voter's baseline belief that a party is honest. Bipartisan scandals, however, do cause the voter to update positively on the aligned party. The fact that the aligned party did not suppress information that it received about the politician's misbehavior makes the voter more inclined to believe that it is the honest type, and hence $\phi_{\text {align }}(1,1)>\gamma$, provided $s>0$.

When exposed to a partisan scandal, the voter is much less certain about the parties' types and the politician's behavior. It is possible that both parties learned about misbehavior but that the aligned party opted to suppress it. Alternatively, neither party may have learned about misbehavior, but the opposing party opted to cast a false accusation. As the next proposition stipulates, the voter's updated beliefs about the politician's behavior and the inferences she makes about the parties both depend upon two key parameters, $\pi$ and $p$.

Proposition 6 Partisan scandals arise only if stakes of an election are reasonably large, $x(1-c)>\gamma \frac{1-\pi p}{1-\pi}$. For those parameters, the reputations of both parties decrease,

$$
\begin{gathered}
\phi_{\text {opp }}(0,1)<\gamma \\
\phi_{\text {align }}(0,1)<\gamma \\
\phi_{\text {opp }}(0,1)+\phi_{\text {align }}(0,1)=\gamma .
\end{gathered}
$$

If voters do not expect scandals, such that $\pi p<\frac{1}{2}$, then

1. $\phi_{\text {opp }}(0,1)<\phi_{\text {align }}(0,1)$;

2. $\Phi(0,1) \geq \pi$.

If voters expect scandals, such that $\pi p>\frac{1}{2}$, then

\footnotetext{
${ }^{19}$ If we interpret $\pi$ as the "the strength of the rumor" and the instigation of a scandal by the aligned party as the active withdrawal of support for one of its members, some inferences about recent scandals follow rather naturally. Consider, for example, the 2017 case of Senator Al Franken being accused of sexual misconduct, and allow $\pi$ to capture the strength of the initial evidence against him. We know that the party that benefits from Franken's collaboration will never pretend to observe misbehavior. The fact that the Democratic Party encouraged Franken to resign, then, should lead the voter to conclude that misbehavior did in fact occur.
} 
3. $\phi_{\text {opp }}(0,1)>\phi_{\text {align }}(0,1)$;

4. $\Phi(0,1) \leq \pi$.

All inequalities are strict if $s, f<1$.

Notice, first, that partisan scandals always damage both parties' reputations. Having observed a partisan scandal, the voter can be sure that one of the two parties is the strategic type; and as a consequence, she becomes more pessimistic about both.

The damage wrought by partisan scandals, however, is not equally distributed across the two parties. Rather, the reputational fallout for each of the parties depends upon the voter's baseline beliefs about the incidence of misbehavior and the probability that the parties learn about it. To understand the intuition for Proposition 6, consider first the case in which $\pi p<\frac{1}{2}$, when parties are unlikely to have information about misbehavior, either because misbehavior is rare or hard to detect. Here, the voter does not expect to see scandals, and so she is inclined to believe that a partisan scandal is triggered by fabrication rather than suppression, causing her to penalize the opposing party more than the aligned one. Knowing the voter's calculus, the opposing party fabricates fewer scandals, but not to the extent that the inference is wiped out. To understand why the implicated politician suffers reputationally, note that the voter's inference from a partisan scandal depends on whether a partisan scandal is more likely when the politician misbehaved or when he did not. The former is higher when suppression $s$ is higher than fabrication $f$, and vice versa. The opposing party appropriately curtails its dishonesty to mitigate the reputational fallout, so indeed suppression is higher than fabrication, $s>f$, and hence $\Phi(0,1) \geq \pi$.

When $\pi p>\frac{1}{2}$, the voter expects that parties are privy to information on misbehavior, and hence she expects a scandal. Under this scenario, the voter is inclined to interpret a partisan scandal as a result of suppression and not fabrication, and she therefore penalizes mainly the aligned party for the perceived dishonesty. The aligned party responds by decreasing suppressions, which leads to $s<f$. This in turn means that the voter expects the aligned party to participate in casting accusations. So when it does not, i.e., when scandal is partisan, the voter takes it as a sign that no misbehavior occurred. Remarkably, then, the politician's reputation benefits from a partisan scandal. 
In this way, we can see how the subjects of political scrutiny can actually benefit from partisan scandal. While both parties suffer reputationally, albeit not equally, the politician himself comes out looking better than he did before. Though hardly dispositive, this finding at least rationalizes a curious feature of contemporary American politics: partisan scandals routinely damage the reputations of both the Democratic and Republican parties, while the public approval ratings of these scandals' primary subject-be he Bill Clinton or Donald Trump-appear noticeably resilient.

The next proposition clarifies how rising the stakes of an election affect the political consequences of partisan scandal.

Proposition 7 As $x(1-c)$ increases, $\left|\phi_{\text {opp }}(0,1)-\phi_{\text {align }}(0,1)\right|$ increases and $|\Phi(0,1)-\pi|$ decreases. ${ }^{20}$

When polarization $x$ rises, the reputational fallout of partisan scandals falls disproportionately on one party, and the consequences of partisan scandals for the politician, whether positive or negative, attenuate. Similarly, and consistent with Hirano and Snyder's (2018) empirical findings on the subject, the political consequences of scandals vary according to a party's entrenchment in a political office, $c$. On net, when stakes of an election are high due to high polarization and low political entrenchment of the aligned party, the difference in political fallout for the parties is large whereas the consequences for the implicated politician tend to be small.

\section{Conclusion}

Details about political scandals intermittently baffle and astound. Often, no rationale would seem to account for the immoral, illegal, or unethical acts at their center. The reasons why politicians do things that endanger their and their associates' careers seem incomprehensible. And perhaps they are. But the occurrence of scandals is not. The transformation of private misbehavior into public scandal is a deeply political process.

\footnotetext{
${ }^{20}$ The comparative statics with respect to $p$ and $\pi$ depends on the parameters in a complicated way that is not instructive.
} 
To investigate this political process, we study a model that is intentionally austere. The model abstracts away from many factors that condition the frequency and consequences of scandal production, such as the partisan leanings of voters (Cortina and Rottinghaus 2017), timing considerations about when to reveal misbehavior (Gratton, Holden, and Kolotilin 2018), the resources and objectives of the media (Entman 2012), the influence of fact-checkers that might independently verify charges of misconduct (Nyhan and Reifler 2015), evolving understandings of political misconduct (Adut 2005, 2008), and the contextual relevance of different types of misbehavior (Nyhan 2015). Future work should investigate these matters. As the first step, however, this paper appropriately focuses on the strategic behaviors of those individuals and parties that stand at a scandal's very center.

Our model yields a rich collection of insights. For example, as the returns from collaboration improve, aligned parties are prone to suppress information about a politician's misbehavior - much as Republicans may have done in our first motivating example. Similarly, higher returns from collaboration also affect the reputational gains from accusing a politician of having misbehaved and the reputational losses from not doing so. And no wonder. When a party discloses the misbehavior of a close associate, as Democrats did in the second motivating example, the voter is especially likely to conclude that it must be the honest type. If it does not do so, following Republicans' example in the first, the voter has reason to conclude that the party knew about the misbehavior all along but opted to stay quiet in order to reap the gains of continued collaboration, as only the strategic type would do.

We also find that polarization accelerates the production of political scandals, a finding that is at once immediately relevant for contemporary American politics and amenable to empirical investigation. Because these scandals tend to be partisan in nature, however, the voter does not learn much about the politician in question. Remarkably, scandals in this setting can redound to the benefit of the implicated politician. When only the opposing party alleges misbehavior, the voter may infer that the politician did not misbehave after all, even as she downgrades her assessment of both parties - a finding, we suggest, that is at least consistent with Trump maintaining steady approval ratings amidst widespread accusations of scandal, while the reputations of Republicans more generally foundered. 
The model also clarifies why higher numbers of scandal do not necessarily imply higher levels of misbehavior. Fixing existing levels of misbehavior, one may observe very different levels of scandals depending on the benefits parties receive from working with a politician, the probability that the parties will learn about his misbehavior, and the likelihood that he will be replaced by someone with different partisan commitments. Moreover, changes in misbehavior do not necessarily yield equivalent changes in scandal. Indeed, marginal increases in misbehavior sometimes decrease the number of scandals that arise. As C. Vann Woodward notes:

It would be misleading to form any first impressions of the relative health or virtue of presidential administrations on the basis of the number of allegations of misconduct filled against them [..] Allegations are not proof, and the volume of allegations may be more an index of the strength of congressional opposition, or the zeal of critics and the austerity of their standards than the culpability of the accused.

The lesson for empirical work is apparent: scandals can be a poor proxy for actual misconduct; and efforts to ascertain the depth of an underlying problem on the basis of public accusations about it can be misleading.

Throughout this paper, we have equated $x$ with polarization. Interpreted in alternative ways, however, the parameter reveals other strategic dimensions of scandal politics. Most obviously, $x$ can be understood as the importance of politician's vote for a legislative outcome. To see this, compare a legislator in a chamber in which one party holds a bare majority and another in which it holds a massive supermajority. Clearly, keeping polarization constant, the value of this legislator is much higher in the former case, and hence, we should expect him to be a more frequent subject of partisan accusations, but also less susceptible to their consequences. Similarly, we might relate $x$ to features of the electoral environment. Compare two electoral settings, one in which the outcome is in doubt and the other in which one party is all but assured to win. In the former environment, parties should value their reputations more, which, indirectly, may be reflected in the assignment of a lower $x$. In this case, we should expect more misbehavior to come to light in the form of bipartisan 
scandals, and partisan scandals to have larger reputational consequences for the implicated politician. Loosely speaking, heightened inter-party competition should encourage voters to hold misbehaving politicians accountable for their actions.

In various ways, the logic of revelation also varies according to the acts and relationships that characterize different scandals. Beyond our motivating examples, the Trump presidency again provides numerous examples of each. Our model captures the logic of scandals that concern acts committed without any obvious consideration for their political consequence, such as Trump's alleged dalliances with porn stars and Playboy models. In the Supplementary Material, we turn our attention to scandals that arise from calculated misbehavior, which broadly characterizes the subject of Robert Mueller's investigations into the Russian government's interactions with the Trump campaign and the House's decision to impeach Trump for withholding military aid for Ukraine. With misbehavior endogenized, we show, most of the main findings about the incidence of scandals and their reputational consequences carry through. Consistent with the finding that polarization and political entrenchment lead to scandals that prevent the voter from correctly evaluating the politician, for instance, we find that such circumstances also encourage politicians to misbehave. Ideological polarization, asa such, does not only breed partisan scandals and voter confusion. It also stimulates misconduct. When conducting empirical work on scandals, therefore, it will not do to simply count their occurrence or measure their general significance. Attention must be paid to the nature of the acts and the structure of relationships between the implicated politicians and associated parties. Scandals are decidedly not idiosyncratic or arbitrary-but nor are they interchangeable. 


\section{Appendix}

\subsection{Preliminaries}

Bayes' rule implies $\phi_{\text {opp }}(1,1)=\gamma, \phi_{\text {align }}(0,0)=\gamma$, and

$$
\begin{gathered}
\phi_{\text {align }}(1,1)=\frac{\gamma}{\gamma+(1-s)(1-\gamma)}, \quad \phi_{\text {opp }}(0,0)=\frac{\gamma}{\gamma+(1-f)(1-\gamma)}, \\
\Phi(1,1)=1, \text { and } \Phi(0,0)=\pi \frac{1-p}{1-\pi p},
\end{gathered}
$$

and if $f+s \neq 0$, then

$$
\begin{gathered}
\phi_{\text {align }}(0,1)=f \gamma \frac{1-\pi p}{f(1-\pi p)+\pi p s}, \quad \phi_{\text {opp }}(0,1)=\pi p \gamma \frac{s}{f(1-\pi p)+\pi p s}, \\
\Phi(0,1)=\pi \frac{f(1-p)+p s}{f(1-\pi p)+\pi p s} .
\end{gathered}
$$

Since the opposing party alleges misbehavior whenever the aligned party does, the incidence of bipartisan scandals is

$$
S_{b i}=\pi p(\gamma+(1-\gamma)(1-s))
$$

The the total number of scandals is

$$
S=f(1-\pi p)(1-\gamma)+\pi p
$$

Using formulas above, the probability that the voter makes a mistake is

$$
\begin{aligned}
M & =\pi \operatorname{Pr}(\text { not fired } \mid m=1)+(1-\pi) \operatorname{Pr}(\text { fired } \mid m=0)= \\
& =2 \pi(1-\pi)\left(\frac{1-p}{1-\pi p}+(1-\pi) p(1-\gamma) \frac{f s}{(1-\pi p)(f(1-\pi p)+\pi p s)}\right)
\end{aligned}
$$

\subsection{Proofs for Section 5}

Notation 1 Let $z \equiv(1-c) x$ denote the stakes of election. 
Proof for Proposition 1. To prove Proposition 1, we prove Lemma 1 below, which describes equilibria in more detail. Those details will be useful in the subsequent proofs.

Lemma 1 Recall $z \equiv(1-c) x$. The following describes all equilibria in which the aligned party never fabricates accusations and the opposing party never suppresses information.

1. There exists a fully honest equilibrium, $f=s=0$, if and only if

$$
z \leq 2 \frac{\gamma(1-p \pi)}{1-\pi}
$$

2. There exists a fully mixing equilibrium in which

$$
\begin{gathered}
s=\frac{(1-\pi) z-\gamma(1-\pi p)}{(1-\gamma)((1-\pi) z+\pi p \gamma)} \\
f=\pi p \frac{(1-\pi) z-\gamma(1-\pi p)}{(1-\gamma)\left(\gamma(\pi p-1)^{2}+\pi p(1-\pi) z\right)},
\end{gathered}
$$

if and only if

$$
\frac{\gamma(1-\pi p)}{(1-\pi)}<z<\min \left\{\frac{1-\pi p \gamma}{1-\pi}, \frac{1-\pi p}{\pi p} \frac{1-\gamma+\pi p \gamma}{1-\pi}\right\}
$$

3. There exists an equilibrium in which $f=1$ and

$$
s=z \frac{1-\pi}{(1-\gamma)(1-\pi) z+\gamma(1-\gamma+\pi p \gamma)},
$$

if and only if $\pi p>\frac{1}{2}$ and $z \in\left(\frac{1-\pi p}{\pi p} \frac{1-\gamma+\pi p \gamma}{(1-\pi)}, \frac{1-\gamma+\pi p \gamma}{1-\pi}\right)$.

4. There exists an equilibrium in which $s=1$ and

$$
f=\pi p z \frac{1-\pi}{\pi p(1-\gamma)(1-\pi) z+\gamma(1-\pi p)(1-\pi p \gamma)},
$$

if and only if $\pi p<\frac{1}{2}$ and $z \in\left(\frac{1-\pi p \gamma}{1-\pi}, \frac{1-\pi p}{\pi p} \frac{1-\pi p \gamma}{1-\pi}\right)$. 
5. There exists a fully dishonest equilibrium, $f=s=1$, if and only if

$$
\max \left\{\frac{(1-\gamma(1-\pi p))}{(1-\pi)}, \frac{1-\pi p \gamma}{1-\pi} \frac{1-\pi p}{\pi p}\right\} \leq z
$$

Proof of Lemma 1. Consider incentives of the aligned party with $v=1$. In equilibria we consider, this party knows that $a_{o p p}=1$, so its payoffs as a function of its decision are:

$$
\begin{array}{ll}
{\left[a_{\text {align }}=1\right] \quad: \quad \phi_{\text {align }}(1,1)+c x} \\
{\left[a_{\text {align }}=0\right] \quad: \quad \phi_{\text {align }}(0,1)+(1-\Phi(0,1)) x+\Phi(0,1) c x}
\end{array}
$$

So it weakly prefers to suppress information if and only if

$$
\phi_{\text {align }}(1,1)-\phi_{\text {align }}(0,1) \leq(1-\Phi(0,1)) z
$$

If $v=0$, the opposing party knows $a_{\text {align }}=0$, so its payoffs as a function of its decision are:

$$
\begin{array}{ll}
{\left[a_{\text {opp }}=1\right]:} & \phi_{\text {opp }}(0,1)-(1-\Phi(0,1)) x-\Phi(0,1) x c \\
{\left[a_{\text {opp }}=0\right]:} & \phi_{\text {opp }}(0,0)-(1-\Phi(0,0)) x-\Phi(0,0) x c
\end{array}
$$

So it weakly prefers to fabricate if and only if

$$
\phi_{\text {opp }}(0,1)-\phi_{\text {opp }}(0,0) \geq-(\Phi(0,1)-\Phi(0,0)) z .
$$

For now, we assume that the aligned party does not fabricate scandals and the opposing party does not suppress information, and consider all possible equilibria under this assumption. At the end of the proof, we show that such behavior can be sustained in equilibrium.

It is immediate to show that if $f=0$, then the opposing party chooses $s=0$, and if $s=0$, then the aligned party chooses $f=0$. Consider then $f=s=0$. In this equilibrium, parties' actions agree, so $\Phi(0,1), \phi_{\text {opp }}(0,1)$ and $\phi_{\text {align }}(0,1)$ are not pinned down by Bayes' rule. But if this is an equilibrium, then using (12) and (13) together with the formulas from 
Section 7.1, the following must hold

$$
\frac{\gamma-\phi_{\text {opp }}(0,1)}{z}+\frac{(1-p) \pi}{(1-p) \pi+(1-\pi)} \geq \Phi(0,1) \geq \frac{-\gamma+\phi_{\text {align }}(0,1)}{z}+1
$$

The left-hand side is largest and the right-hand side smallest when $\phi_{\text {opp }}(0,1)=\phi_{\text {align }}(0,1)=$ 0 . So the existence of this equilibrium requires

$$
\frac{\gamma}{z}+\frac{(1-p) \pi}{(1-p) \pi+(1-\pi)} \geq \Phi(0,1) \geq \frac{-\gamma}{z}+1
$$

and hence we can find a nonempty set of $\Phi(0,1) \in(0,1)$ if and only if (5) is satisfied.

Consider now an equilibrium in which both parties mix: $f \in(0,1)$ and $s \in(0,1)$. Plugging formulas from Section 7.1 into (12) and (13) satisfied with equalities, and solving for $f$ and $s$, we obtain (6) and (7). For this to be an equilibrium, we need that indeed $f \in(0,1)$ and $s \in(0,1)$, which delivers $(8)$.

Consider now $f=1$ but $s \in(0,1)$. From (12) and (13), this equilibrium requires

$$
\begin{gathered}
\phi_{\text {align }}(1,1)-\phi_{\text {align }}(0,1)=(1-\Phi(0,1)) z, \\
\phi_{\text {opp }}(0,1)-\phi_{\text {opp }}(0,0) \geq-(\Phi(0,1)-\Phi(0,0)) z .
\end{gathered}
$$

Plugging formulas from Section 7.1, we obtain that (9) solves the first equation and the inequality is satisfied if $z \geq \frac{(1-\pi p)}{\pi p} \frac{-\gamma+\pi p \gamma+1}{(1-\pi)}$. Condition $s \in(0,1)$ requires that $\frac{1-\gamma+\pi p \gamma}{(1-\pi)}>z$. Combining these, we obtain the condition of part 2 of the lemma.

Consider now $s=1$ and $f \in(0,1)$. From (12) and (13), this equilibrium requires

$$
\begin{gathered}
\phi_{\text {align }}(1,1)-\phi_{\text {align }}(0,1) \leq(1-\Phi(0,1)) z, \\
\phi_{\text {opp }}(0,1)-\phi_{\text {opp }}(0,0)=-(\Phi(0,1)-\Phi(0,0)) z .
\end{gathered}
$$

Plugging the formulas from Section 7.1, we obtain that (10) solves the second equation and the inequality is satisfied if $z \geq \frac{1-\pi p \gamma}{(1-\pi)}$. Condition $f \in(0,1)$ requires that $z<(1-\pi p) \frac{1-\pi p \gamma}{\pi p(1-\pi)}$. Combining these, we obtain the condition of part 3 of the lemma. 
Consider now $f=1$ and $s=1$. From (12) and (13), this is an equilibrium if and only if

$$
\begin{gathered}
\phi_{\text {align }}(1,1)-\phi_{\text {align }}(0,1) \leq(1-\Phi(0,1)) z, \\
\phi_{\text {opp }}(0,1)-\phi_{\text {opp }}(0,0) \geq-(\Phi(0,1)-\Phi(0,0)) z .
\end{gathered}
$$

Plugging formulas from Section 7.1 and using $f=s=1$, we obtain that this is an equilibrium if and only if (11) holds.

It remains to show that in any putative equilibrium identified above, the aligned party has no incentive to fabricate a scandal and the opposing party has no incentive to suppress information. Consider the aligned party. If no misbehavior is observed, its payoffs as a function of its decision are:

$$
\begin{aligned}
{\left[a_{\text {align }}=1\right]: \quad } & f\left[\phi_{\text {align }}(1,1)+c x\right] \\
& +(1-f)\left[\phi_{\text {align }}(1,0)+(1-\Phi(1,0)) x+\Phi(1,0) c x\right] \\
{\left[a_{\text {align }}=0\right]: \quad } & f\left[\phi_{\text {align }}(0,1)+(1-\Phi(0,1)) x+\Phi(0,1) c x\right] \\
& +(1-f)\left[\phi_{\text {align }}(0,0)+(1-\Phi(0,0)) x+\Phi(0,0) c x\right] .
\end{aligned}
$$

So it does not fabricate if

$$
\begin{gathered}
f\left(\left(\phi_{\text {align }}(1,1)-\phi_{\text {align }}(0,1)\right)-(1-\Phi(0,1)) z\right) \\
+(1-f)\left(\phi_{\text {align }}(1,0)-\phi_{\text {align }}(0,0)-z(\Phi(1,0)-\Phi(0,0))\right) \leq 0 .
\end{gathered}
$$

The first expression in (14) is negative if $f>0$ and is 0 if $f=0$. So using $\phi_{\text {align }}(1,1)=\gamma$ from Section 7.1, (14) is satisfied if

$$
\phi_{\text {align }}(1,0)-\gamma-z\left(\Phi(1,0)-\pi \frac{1-p}{1-\pi p}\right) \leq 0
$$

Consider the opposing party. If misbehavior is observed, its payoffs as a function of its 
decision are:

$$
\begin{aligned}
{\left[a_{\text {opp }}=1\right]: } & s\left(\phi_{\text {opp }}(0,1)-(1-\Phi(0,1)) x-\Phi(0,1) x c\right) \\
& +(1-s)\left(\phi_{\text {opp }}(1,1)-x c\right) ; \\
{\left[a_{\text {opp }}=0\right]:=} & s\left(\phi_{\text {opp }}(0,0)-(1-\Phi(0,0)) x-\Phi(0,0) x c\right) \\
+ & +(1-s)\left(\phi_{\text {opp }}(1,0)-(1-\Phi(1,0)) x-\Phi(1,0) x c\right) .
\end{aligned}
$$

So it prefers to not to suppress information if

$$
\begin{aligned}
& s\left(\phi_{\text {opp }}(0,1)-\phi_{\text {opp }}(0,0)+(\Phi(0,1)-\Phi(0,0)) z\right) \\
+ & (1-s)\left(\phi_{\text {opp }}(1,1)-\phi_{\text {opp }}(1,0)+(1-\Phi(1,0)) z\right) \geq 0 .
\end{aligned}
$$

The first expression in (16) is positive if $s>0$ and is 0 if $s=0$. So using $\phi_{\text {opp }}(0,0)=\gamma$ from Section 7.1, (16) is satisfied if

$$
\gamma-\phi_{\text {opp }}(1,0)+(1-\Phi(1,0)) z \geq 0
$$

Since history $(1,0)$ is off the equilibrium path, Bayes rule does not restrict $\phi_{\text {opp }}(1,0), \phi_{\text {align }}(1,0)$ and $\Phi(1,0)$, and straightforwardly one can find such beliefs that satisfy (15) and (17). E.g., $\phi_{\text {opp }}(1,0)=\phi_{\text {align }}(1,0)=\gamma$ and $\Phi(1,0) \in\left(\pi \frac{1-p}{1-\pi p}, 1\right)$ suffice. Hence, the behavior described in the lemma constitutes equilibria for certain off-equilibrium beliefs.

\subsection{Proofs for Section 5.1}

Proof of Proposition 2. With our equilibrium selection, $f$ and $s$ are absolutely continuous $^{21}$. Hence, to establish any comparative statics of Propositions 2, 3 and 4 on $f$ and $s$, it is sufficient to establish that this comparative statics holds within each equilibrium identified in Lemma 1, though looking across equilibria is required when proving single-peakedness of $f$ in Proposition 4. The comparative statics on $f$ (and $s$ ) is trivially true for the set of parameters for which $f=0$ or $f=1$ ( $s=0$ or $s=1$ ), so it suffices to focus on the remaining

\footnotetext{
${ }^{21} \mathrm{~A}$ formal proof is straightforward and available from the authors upon request.
} 
equilibria types, which are three: full randomization, $f=1$ and $s<1$, and $f<1$ and $s=1$.

Recall that $z$ denotes stakes of election. Totally differentiating (6), (7), (9), and (10) with respect to $z$, we obtain that $f$ and $s$ are always weakly increasing in $z$, and strictly so whenever $f \in(0,1)$ and $s \in(0,1)$, respectively. From $(2), z$ affects the incidence of bipartisan scandals only via $s$, it decreases in $s$, and by what precedes, $s$ increases in $z$, so $S_{b i}$ decreases in $z$. From (3), $z$ affects the incidence of all scandals only via $f$, and by what precedes, $f$ increases in $z$, hence $S$ increases in $z$. And finally, differentiating voter's probability of making mistake (4) with respect to $z$ we obtain

$$
\frac{d M}{d z}=2 \pi(1-\pi)^{2} p(1-\gamma) \frac{\frac{d f}{d z} \pi p s^{2}+f^{2}(1-\pi p) \frac{d s}{d z}}{(1-\pi p)(f(1-\pi p)+\pi p s)^{2}}
$$

so by the fact that $f$ and $s$ increase in $z, \frac{d M}{d z} \geq 0$ in every equilibrium.

\subsection{Proofs for Section 5.2}

Proof of Proposition 3. Totally differentiating (7) and (10) with respect to $p$ we obtain $\frac{d f}{d p}>0$. Totally differentiating (6) with respect to $p$, we obtain $\frac{d s}{d p}>0$, and totally differentiating (9) with respect to $p$, we obtain $\frac{d s}{d p}<0$. From (2), $p$ affects $S_{b i}$ directly and via s. $S_{b i}$ increases in $p$ and decreases in $s$, and by what precedes, $s$ decreases in $p$ when $f=1$, so $S_{b i}$ increases in $p$ when $f=1$, or $s=0$, or $s=1$. Totally differentiating (2) and using (6), we obtain that when the equilibrium is fully mixing, $\frac{d S_{b i}}{d p}=\pi z \gamma \frac{1-\pi}{(z-\pi z+\pi p \gamma)^{2}}>0$. From (3), $p$ affects $S$ directly and via $f$. Also, $S$ strictly increases in $p$ and weakly in $f$, and from part (1), $f$ increases in $p$, so $S$ strictly increases in $p$. From (4), $\frac{d M}{d p}<0$ when $f=s=0$. In the fully mixing equilibrium, using (6) and (7) in (4), we obtain $M=2 \pi(1-\pi) \frac{z(1-\pi)+\gamma(1-2 p+\pi p)}{z(1-\pi)+\gamma(1-\pi p)}$, so $\frac{d M}{d p}<0$. When $s=1$ and $f<1$, plugging $s=1$ and (10) into (4), we obtain $M=$ $2 \pi(1-\pi) \frac{(\pi p \gamma-p \gamma-\pi+1) z+\left(\pi p^{2} \gamma^{2}-\pi p \gamma^{2}-p \gamma+\gamma\right)}{(1-\pi p \gamma)(z(1-\pi)+\gamma(1-\pi p))}$, so

$$
\frac{d M}{d p}=-2 \pi(1-\pi)^{2} \gamma \frac{(\pi-1)^{2} z^{2}+(1-\pi)(1+\gamma-2 \pi p \gamma) z+\gamma(\pi p \gamma-1)^{2}}{((1-\pi p \gamma)(z(1-\pi)+\gamma(1-\pi p)))^{2}}<0
$$

where the inequality follows from Lemma 1, which says that this equilibrium requires $2 p \pi<1$. When $s<1$ and $f=1$, then plugging $f=1$ and (6) into (4), we obtain 
$M=2 \pi(1-\pi)\left(\frac{1-p}{1-\pi p} \gamma+(1-\gamma) \frac{1-p+p s}{1-\pi p+\pi p s}\right)$, so

$\frac{d M}{d p}=2 \pi(1-\pi)\left(\frac{-(1-\pi)}{(1-\pi p)^{2}} \gamma-(1-\gamma) \frac{(1-s)(1-\pi)}{(1-\pi p+\pi p s)^{2}}+(1-\gamma) \frac{p(-\pi+1)}{(1-\pi p+\pi p s)^{2}} \frac{d s}{d p}\right)<0$

where we use the fact that $\frac{d s}{d p}<0$ when $f=1$.

\subsection{Proofs for Section $\mathbf{5 . 3}$}

Proof of Proposition 4. Totally differentiating (6) and (9), we obtain $\frac{d s}{d \pi}<0$. Totally differentiating (7), we obtain

$$
\frac{d f}{d \pi}=\frac{p \gamma}{(1-\gamma)} \frac{p(z-p \gamma) \pi^{2}+(2 p \gamma-2 z) \pi+(z-\gamma)}{\left(\gamma\left(1+\pi^{2} p^{2}-2 \pi p\right)+\pi p z(1-\pi)\right)^{2}}\left\{\begin{array}{c}
>0 \text { if } \pi<\pi_{0} \\
<0 \text { if } \pi>\pi_{0}
\end{array},\right.
$$

where

$$
\pi_{0} \equiv \frac{1}{p(z-p \gamma)}(z-p \gamma-\sqrt{z(z-p \gamma)(1-p)}) \in(0,1)
$$

Totally differentiating (10), we obtain

$$
\frac{d f}{d \pi}=p \gamma z \frac{p(\gamma-p \gamma+1) \pi^{2}-2 \pi+1}{(\pi p(1-\gamma)(1-\pi) z+\gamma(1-\pi p)(1-\pi p \gamma))^{2}}\left\{\begin{array}{c}
>0 \text { if } \pi<\pi_{1} \\
<0 \text { if } \pi>\pi_{1}
\end{array},\right.
$$

where

$$
\pi_{1} \equiv \frac{1}{p} \frac{1-\sqrt{(p \gamma-1)(p-1)}}{\gamma-p \gamma+1} .
$$

Note that $\pi_{1}$ is independent of $z$, while $\pi_{0}$ increases in $z$ for $z>\gamma$, and $\lim _{z \rightarrow \infty} \pi_{0}=$ $\frac{1}{p}(1-\sqrt{(1-p)})<\pi_{1}$, so $\pi_{0}<\pi_{1}$ for $z>\gamma$.

From Lemma 1, when $z<\gamma$, then in all equilibria, $f=0$, so the proposition is satisfied trivially. Consider any $z>\gamma$. Since equilibrium $f$ is continuous in $\pi$ and $\pi_{0}<\pi_{1}$, the only way for $f$ not to be single-peaked is if for some $\pi \in\left(\pi_{0}, \pi_{1}\right)$, the equilibrium switches from the fully mixing one (so $\mathrm{f}$ is decreasing in $\pi$ at that point) to the one with $s=1$ and $f<1$ (so $\mathrm{f}$ is increasing in $\pi$ at that point). But this cannot happen. To see that, take $z$ that satisfies (8) evaluated at $\pi_{0}$, so the equilibrium is fully mixed at $\pi_{0}$. As $\pi$ increases, as long 
as $\pi p<\frac{1}{2}$, then both the lower and the upper bound of the interval (8) increase, so the equilibrium moves to $s=f=0$, where is stays for all higher $\pi$. Suppose then that $\pi p>\frac{1}{2}$ at some point. But then by Lemma 1, the equilibrium with $s=1, f<1$ does not exist.

From (2), $\pi$ affects $S_{b i}$ directly and via $s$, and $S_{b i}$ increases in $\pi$ and decreases in $s$, so since $s$ decreases in $\pi$, then $S_{b i}$ increases in $\pi$. From (3), $\pi$ affects $S$ directly and via $f$. Since the direct effect of $\pi$ on $S$ is positive, $S$ increases in $\pi$ as long as $f$ increases in $\pi$. So to prove part (3) of Proposition 3 it suffices to show that $S$ may decrease for intermediate values of $z=x(1-c)$. To do so, consider the fully mixing equilibrium. Totally differentiating $S$ with respect to $\pi$ and using (18) and (7), we obtain

$$
\begin{aligned}
\frac{d S}{d \pi} & =p(1-(1-\gamma) f)+(1-\pi p)(1-\gamma) \frac{d f}{d \pi} \\
& =p z \gamma(1-\pi p) \frac{1-2 \pi+\pi p}{\left(\gamma+\pi^{2} p^{2} \gamma-2 \pi p \gamma-\pi^{2} p z+\pi p z\right)^{2}}\left\{\begin{array}{c}
>0 \text { for } \pi<\frac{1}{2-p} \\
<\text { for } \pi>\frac{1}{2-p}
\end{array}\right.
\end{aligned}
$$

Consider $z$ in the interior of the interval (8) from part 4 of Lemma 1 evaluated at $\pi=\frac{1}{2-p}$, that is $z \in\left(2 \gamma, \min \left\{\frac{2-p-p \gamma}{1-p}, 2 \frac{-p-2 \gamma+2 p \gamma+2}{p}\right\}\right)$. By continuity, this $z$ is still in the interval (8) for some $\pi$ greater than $\frac{1}{2-p}$, so for some $\pi$ greater than $\frac{1}{2-p}$, the equilibrium is fully mixing and by what proceeds, $\frac{d S}{d \pi}<0$.

\subsection{Proofs for Section 5.4}

Proof of Proposition 5. Follows directly from the formulas in Section 7.1.

Proof of Proposition 6. Partisan scandals arise only if neither of the parties is fully honest, which from Lemma 1 is when $z>\gamma \frac{1-\pi p}{1-\pi}$. From Section 7.1, then $\phi_{\text {align }}(0,1)<\gamma$ and $\phi_{\text {opp }}(0,1)<\gamma$, and $\phi_{\text {align }}(0,1)+\phi_{\text {opp }}(0,1)=\gamma$, and $\phi_{\text {opp }}(0,1)<\phi_{\text {align }}(0,1)$ if and only if

$$
\frac{\pi p}{1-\pi p}<\frac{f}{s}
$$

Consider first the fully mixing equilibrium. Using (6) and (7), inequality (20) is satisfied when $\pi p<\frac{1}{2}$ and violated when $\pi p>\frac{1}{2}$. When $\pi p>\frac{1}{2}$, we also may have equilibria with $(s \leq 1, f=1)$, and using (9) we obtain that inequality (20) is violated if $z>$ 
$\frac{(1-\pi p)(1-\gamma+\pi p \gamma)}{(1-\pi) \pi p} \frac{\gamma \pi p}{(\gamma+2 \pi p-\pi p \gamma-1)}$, which is always satisfied for $z>\frac{(1-\pi p)(1-\gamma+\pi p \gamma)}{(1-\pi) \pi p}$, which is a perquisite for this equilibrium. When $\pi p<\frac{1}{2}$, we also may have equilibria with $(s=1, f \leq 1)$, and using (10) we obtain that inequality (20) is satisfied if $\frac{(1-\pi p \gamma)}{(1-\pi)} \frac{\gamma(1-\pi p)}{(-2 \pi p+\pi p \gamma+1)}<z$, which is always satisfied for $\frac{(1-\pi p \gamma)}{(1-\pi)}<z$, which is a perquisite for this equilibrium.

Using the formula for $\Phi(0,1)$, we obtain $\Phi(0,1)>\pi$ if and only if $f<s$. This is true in equilibrium $(s=1, f<1)$, which can arise if and only if $\pi p<\frac{1}{2}$, and is violated in equilibrium $(s<1, f=1)$, which can arise if and only if $\pi p>\frac{1}{2}$. In fully mixing equilibrium, using (6) and (7) we can establish that this is true also if and only if $\pi p<\frac{1}{2}$. And in $(s=f=1)$ equilibrium, we obtain $\Phi(0,1)=\pi$.

\section{Proof for Proposition 7.}

From Proposition 6 , since $\phi_{\text {align }}(0,1)+\phi_{\text {opp }}(0,1)=\gamma,\left|\phi_{\text {opp }}(0,1)-\phi_{\text {align }}(0,1)\right|=2 \phi_{\text {opp }}(0,1)-$ $\gamma$ if $p \pi>\frac{1}{2}$ and $\left|\phi_{o p p}(0,1)-\phi_{\text {align }}(0,1)\right|=\gamma-2 \phi_{\text {opp }}(0,1)$ if $p \pi<\frac{1}{2}$. So the comparative statics with respect to $z$ holds if $\phi_{\text {opp }}(0,1)$ decreases in $z$ for $p \pi<\frac{1}{2}$ and increases in $z$ for $p \pi>\frac{1}{2}$. In the fully mixing equilibrium,

$$
\frac{d \phi_{o p p}(0,1)}{d z}=\pi p \gamma(1-\pi p) \frac{\frac{d s}{d z} f-\frac{d f}{d z} s}{(f(1-\pi p)+\pi p s)^{2}}
$$

which using (6) and (7) can be rewritten as

$\frac{d \phi_{o p p}(0,1)}{d z}=\frac{(\pi p \gamma)^{2}(1-\pi)^{2}(z-\gamma-\pi z+\pi p \gamma)^{2}(2 \pi p-1)}{(f(1-\pi p)+\pi p s)^{2}(\gamma-1)^{2}\left(\gamma+\pi^{2} p^{2} \gamma-2 \pi p \gamma-\pi^{2} p z+\pi p z\right)^{2}(z-\pi z+\pi p \gamma)^{2}}$

so the required comparative statics holds. For the equilibrium with $s<1$ and $f=1$, which can arise only if $\pi p>\frac{1}{2}, \frac{d \phi_{o p p}(0,1)}{d z}$ has the same sign as $\frac{d s}{d z}$, which by Proposition 2 is positive. For the equilibrium with $s=1$ and $f<1$, which can arise only if $\pi p<\frac{1}{2}$, $\frac{d \phi_{o p p}(0,1)}{d z}$ has the same sign as $-\frac{d f}{d z}$, which by Proposition 2 is negative.

From Proposition 6, when $\pi p<\frac{1}{2},|\Phi(0,1)-\pi|=\Phi(0,1)-\pi$, so $|\Phi(0,1)-\pi|$ decreases when $\Phi(0,1)$ decreases. When $\pi p>\frac{1}{2},|\Phi(0,1)-\pi|=\pi-\Phi(0,1)$, so $|\Phi(0,1)-\pi|$ decreases when $\Phi(0,1)$ increases. Totally differentiating $\Phi(0,1)$ from Section 7.1 , we obtain

$$
\frac{d \Phi(0,1)}{d z}=\pi p(1-\pi) \frac{f \frac{d s}{d z}-\frac{d f}{d z} s}{(f(1-\pi p)+\pi p s)^{2}}
$$


Comparing this to $(21)$, the signs of $\frac{d \Phi(0,1)}{d z}$ and $\frac{d \phi_{o p p}(0,1)}{d z}$ are identical so the comparative statics follows.

\subsection{Extension: Endogenous Misbehavior}

We now endogenize misbehavior. The game proceeds as before, except that now the politician, rather than nature, chooses $m$ in the first stage. Suppose that the politician receives benefit $b$ from misbehavior, where $b \sim U[-(1-B), B]$ with $B \in(p, 1)$, and benefit 1 from being in office. ${ }^{22}$ Given the voter's beliefs about incidence of misbehavior $\pi$, the politician with a particular $b$ decides whether to misbehave or not, which determines the actual probability of misbehavior $\pi$. An equilibrium, therefore, identifies the optimal incidence of misbehavior, $\pi^{*}$, given the voter's beliefs are $\pi^{*}$.

Proposition 8 There exists an equilibrium. If there exist multiple equilibria, for each set of parameters consider equilibria with the lowest (highest) equilibrium level of misbehavior $\pi^{*}$. Then

1. $\pi^{*}$ increases in $x(1-c)$;

2. $\pi^{*}$ decreases in $p$.

Proof of Proposition 8. The payoff of the politician that does not misbehave is

$$
(\gamma+(1-\gamma)(1-f))(1-\Phi(0,0))+(1-\gamma) f(1-\Phi(0,1))
$$

and the payoff of the politician that misbehaves is

$b+(1-p)((\gamma+(1-\gamma)(1-f))(1-\Phi(0,0))+(1-\gamma) f(1-\Phi(0,1)))+p(1-\gamma) s(1-\Phi(0,1))$.

So the politician engages in misbehavior if and only if

$$
b \geq p[(\gamma+(1-\gamma)(1-f))(1-\Phi(0,0))+(1-\gamma)(1-\Phi(0,1))(f-s)] .
$$

\footnotetext{
${ }^{22}$ Assuming $B<1$ rules out equilibria in which all politicians misbehave. We assume $B>p$ to ascertain that some types misbehave for most parameters.
} 
Consider first a putative equilibrium in which both parties are honest, $f=s=0$. Then (22) becomes $b \geq p(1-\Phi(0,0))=p\left(1-\pi \frac{1-p}{1-\pi p}\right)$, so the equilibrium incidence of misbehavior is a solution to

$$
\pi=B-p \frac{1-\pi}{1-\pi p} \equiv R H S_{h}(\pi)
$$

There is a unique solution to (23) satisfying $\pi \in[0,1]$, and let us call this solution $\pi_{h}$. Note that $R H S_{h}(\pi=0)=B-p>0$ and $R H S_{h}(\pi=1)=B<1$, so the right-hand side of (23) crosses the left-hand side from above. This, together with $\frac{\partial R H S_{h}(\pi)}{\partial p}<0$ and $\frac{\partial R H S_{h}(\pi)}{\partial z}=0$, implies $\frac{d \pi_{h}}{d p}<0$ and $\frac{d \pi_{h}}{d z}=0$. By Lemma $1, \pi_{h}$ and $f=s=0$ constitute an equilibrium if and only if $z \leq 2 \frac{\gamma\left(1-p \pi_{h}\right)}{\left(1-\pi_{h}\right)}$.

Let $\bar{s}$ and $\bar{f}$ describe the equilibrium $s$ and $f$ identified in Lemma 1 using our equilibrium selection criterium of choosing the equilibrium with larger degree of dishonesty. For any equilibrium other than the honest equilibrium, plugging the formulas for $\Phi(0,0)$ and $\Phi(0,1)$ into (22), we obtain that the politician engages in misbehavior if and only if

$$
b \geq p(1-\pi) \frac{\bar{f}(\gamma+(1-\bar{s})(1-\gamma))+\pi p(\bar{s}-\bar{f})}{(1-\pi p)(\bar{f}(1-\pi p)+\pi p \bar{s})} .
$$

So the incidence of misbehavior solves the following equation

$$
\pi=B-p(1-\pi) \frac{\bar{f}(\gamma+(1-\bar{s})(1-\gamma))+\pi p(\bar{s}-\bar{f})}{(1-\pi p)(\bar{f}(1-\pi p)+\pi p \bar{s})} \equiv \operatorname{RHS}(\pi)
$$

Note that $\operatorname{RHS}(\pi=1)=B<1$ and $\operatorname{RHS}(\pi=0)=B-p(\gamma+(1-\bar{s}(\pi=0))(1-\gamma))>$ $0,{ }^{23}$ and since $\bar{f}$ and $\bar{s}$ are continuous functions of $\pi$ (Lemma 1), RHS $(\pi)$ is a continuous function in $\pi$ mapping $[0,1]$ into $[0,1]$. By Theorem 1 of Villas-Boas (1997), the smallest and the largest fixed points of (24) increase in $z$ and decrease in $p$ if $R H S(\pi)$ increases in $z$ and decreases in $p$. Since $\bar{f}$ and $\bar{s}$ are absolutely continuous in $p$ and $z$, so $R H S(\pi)$ is absolutely continuous in $p$ and $z$, and hence to establish that $R H S(\pi)$ increases in $z$ and decreases in $p$, it suffices to establish that $\frac{\partial R H S(\pi)}{\partial z} \geq 0$ and $\frac{\partial R H S(\pi)}{\partial p}<0$ whenever $\bar{f}$ and $\bar{s}$

\footnotetext{
${ }^{23}$ Note that for $\pi=0, s>0$ as long as $z>\gamma$, but that must be true in any not honest equilibrium.
} 
are differentiable. By Proposition 2, $\bar{f}$ and $\bar{s}$ are weakly increasing in $z$, and

$$
\begin{aligned}
& \frac{\partial R H S(\pi)}{\partial \bar{f}}=p(1-\pi) \frac{\pi p \bar{s}^{2}(2-\gamma)}{(1-\pi p)(\bar{f}(1-\pi p)+\pi p \bar{s})^{2}}>0, \\
& \frac{\partial R H S(\pi)}{\partial \bar{s}}=p(1-\pi) \frac{\bar{f}^{2}(1-\gamma)(1-\pi p)}{(1-\pi p)(\bar{f}(1-\pi p)+\pi p \bar{s})^{2}}>0,
\end{aligned}
$$

so $R H S(\pi)$ indeed increases in z. Moreover, comparing (23) with (24), we see that for any $\pi, \operatorname{RHS}(\pi)>\operatorname{RH} S_{h}(\pi)$ whenever $\bar{f}$ and $\bar{s}>0$, so any solution of (24) that constitutes an equilibrium (that is, delivers $\bar{f}$ and $\bar{s}>0$ ) will be higher than $\pi_{h}$.

To prove part (2), we need to establish that $\frac{\partial R H S_{h}(\pi)}{\partial p}<0$ and $\frac{\partial R H S(\pi)}{\partial p}<0$ whenever $R H S(\pi)$ is differentiable in $p$. Differentiating (23) with respect to $p$ we obtain $\frac{\partial R H S_{h}(\pi)}{\partial p}=$ $-\frac{1-\pi}{(1-\pi p)^{2}}<0$. Consider now a putative equilibrium in which both parties are mixing. Plugging (3) and (4) into (24), we obtain that the equilibrium $\pi$ solves

$$
\pi=B-2 p \gamma \frac{1-\pi}{z(1-\pi)+(1-\pi p) \gamma}
$$

so for the range of parameters for which this equilibrium exist, $\frac{\partial R H S(\pi)}{\partial p}<0$. Consider now a putative equilibrium with $f=1$ and $s<1$. Plugging $f=1$ and (6) into (24), we obtain

$$
\pi=B-p(1-\pi) \gamma \frac{\pi p(1-\pi) z+(1-\pi p)(1-\gamma(1-\pi p))}{(1-\pi p)(-\gamma+\pi p \gamma+1)(z(1-\pi)+\gamma(1-\pi p))}
$$

Differentiating the right-hand side, we obtain that for the range of parameters for which this equilibrium exists,

$$
\begin{aligned}
\frac{\partial R H S(\pi)}{\partial p}= & \frac{-(1-\pi)^{3} \gamma \pi p(2(1-\pi p)(1-\gamma)+\pi p)}{((1-\pi p)(-\gamma+\pi p \gamma+1)(z+\gamma-\pi z-\pi p \gamma))^{2}} z^{2} \\
& -\frac{(1-\pi)^{2} \gamma(1-\pi p)\left(2 p^{2} \gamma(2 \gamma-1) \pi^{2}+p(5 \gamma-1)(1-\gamma) \pi+(\gamma-1)^{2}\right)}{((1-\pi p)(-\gamma+\pi p \gamma+1)(z+\gamma-\pi z-\pi p \gamma))^{2}} z \\
& -\frac{(1-\pi) \gamma^{2}(\pi p-1)^{2}(-\gamma+\pi p \gamma+1)^{2}}{((1-\pi p)(-\gamma+\pi p \gamma+1)(z+\gamma-\pi z-\pi p \gamma))^{2}}
\end{aligned}
$$

which is negative as all coefficients in this quadratic equation are negative. ${ }^{24}$ Consider now

${ }^{24}$ To see that $2 p^{2} \gamma(2 \gamma-1) \pi^{2}+p(5 \gamma-1)(1-\gamma) \pi+(\gamma-1)^{2}>0$, note that this is positive if $2 \gamma>1$, 
a putative equilibrium with $s=1$ and $f<1$. Plugging $s=1$ and (7) into (24), we obtain

$$
\pi=B-p \gamma(z(1-\pi)-\pi p \gamma+1) \frac{1-\pi}{(1-\pi p \gamma)(z(1-\pi)+\gamma(1-\pi p))}
$$

Differentiating the right-hand side, we obtain that for the range of parameters for which this equilibrium exists,

$$
\frac{\partial R H S(\pi)}{\partial p}=-\gamma(1-\pi) \frac{(\pi-1)^{2} z^{2}+(1-\pi)(1+\gamma-2 \pi p \gamma) z+\gamma(\pi p \gamma-1)^{2}}{((1-\pi p \gamma)(z(1-\pi)+\gamma(1-\pi p)))^{2}}<0
$$

where the last inequality follows from the fact that all coefficients in the quadratic equation in the numerator are positive, so this quadratic equation must be positive for any $z>0$. And finally, consider $f=s=1$. Then (24) becomes $\pi=B-p \gamma \frac{1-\pi}{1-\pi p}$, so again $\frac{\partial R H S(\pi)}{\partial p}$.

As the stakes of an election $x(1-c)$ increase, parties are more likely to either fabricate or suppress information. Consequentially, voters' decisions are less informed about the politician's actual misconduct, which encourages the politician to misbehave. The comparative statics with respect to misconduct discoverability $p$ are more involved. From Proposition 3 we know that parties are more likely to act deceptively as their probability of learning about misbehavior increases, and as a result, scandals are less informative about politician misconduct. This gives the politician less incentive to refrain from misbehavior. But parties are also more likely to observe misbehavior when it occurs, so the voter is also more likely to become informed. Proposition 8 states that this latter effect dominates. As $p$ increases, voters' decisions are more closely related to misbehavior, and the politician's incentives to misbehave accordingly decline.

The comparative statics on scandal incidence is more nuanced. From Propositions 2 and 3 we know that as stakes of an election $x(1-c)$ and misconduct discoverability $p$ increase, the incidence of scandals increases via an increase in fake accusations $f$. Having endogenized misbehavior, however, an additional effect comes into play via equilibrium incidence of misbehavior $\pi^{*}$, and from Proposition 4 we know that this effect has an ambiguous sign. As a result, the total effects of $x(1-c)$ and $p$ on the incidence of scandals also are ambiguous. Still, we have the next proposition.

and if $2 \gamma<1$, then this concave quadratic equation is positive for $\pi=0$ and $\pi=1$, so it is positive for all $\pi$. 
Proposition 9 There exist parameters for which the incidence of scandals increases in $p$.

Proof of Proposition 9. Suppose we are in fully mixing equilibrium. Plugging (3) and (4) into (17), we obtain

$$
S=\pi^{*} p z \frac{1-\pi^{*}}{\gamma+\left(\pi^{*}\right)^{2} p^{2} \gamma-2 \pi^{*} p \gamma-\left(\pi^{*}\right)^{2} p z+\pi^{*} p z} .
$$

Totally differentiating $S$ with respect to $p$, we obtain

$$
\frac{d S}{d p}=\frac{\gamma\left(1-\pi^{*} p\right) z\left(\pi^{*}\left(1-\pi^{*}\right)\left(1+\pi^{*} p\right)+p\left(1-(2-p) \pi^{*}\right) \frac{d \pi^{*}}{d p}\right)}{\left(\gamma+\left(\pi^{*}\right)^{2} p^{2} \gamma-2 \pi^{*} p \gamma+\pi^{*} p z\left(1-\pi^{*}\right)\right)^{2}}
$$

Since from the proof of Proposition 8, in the fully mixing equilibrium $\frac{d \pi^{*}}{d p}<0$, a sufficient condition for $\frac{d S}{d p}>0$ is that $\pi^{*}>\frac{1}{2-p}$. From the proof of Proposition 8 we also know that the misbehavior incidence $\pi^{*}$ in the fully mixing equilibrium is higher than the misbehavior incidence in the fully honest equilibrium $\pi_{h}$, so a sufficient condition for $\frac{d S}{d p}>0$ is $\pi_{h}>\frac{1}{2-p}$, which requires $\frac{1}{2 p}\left(B p+1-p-\sqrt{p^{2} B^{2}-2 p(p+1) B+\left(5 p^{2}-2 p+1\right)}\right)>\frac{1}{2-p}$, which in turn requires that $B>\frac{2 p-p^{2}+2}{2(2-p)}$, and this is possible as long as the right-hand side is smaller than 1 , which is true for $p<2-\sqrt{2}$. So it remains to show that the fully mixing equilibrium can exist for $B>\frac{2 p-p^{2}+2}{2(2-p)}$. To show that, first note that the solution to (24) in the fully mixing equilibria is

$$
\pi_{r}(z)=\frac{1}{2 z+2 p \gamma}(z(1+B)+\gamma-2 p \gamma+B p \gamma-\sqrt{\Delta})
$$

where

$\Delta=(B-1)^{2} z^{2}+2 \gamma(B-1)(-2 p+B p-1) z+\gamma^{2}\left(-4 p-4 B p^{2}+B^{2} p^{2}-2 B p+12 p^{2}+1\right)$.

By Lemma 1, it is sufficient to show that there exists $z^{\prime}$ such that $\frac{\gamma\left(1-\pi_{r}\left(z^{\prime}\right) p\right)}{\left(1-\pi_{r}\left(z^{\prime}\right)\right)}=z^{\prime}$ and $\frac{\gamma\left(1-\pi_{r}(z) p\right)}{\left(1-\pi_{r}(z)\right)}<z$ for all $z>z^{\prime}$. Using (26), we see that $\frac{\gamma\left(1-\pi_{r}(z) p\right)}{\left(1-\pi_{r}(z)\right)} \leq z$ if and only if

$$
(B-1) z^{2}-\gamma(5 p-3) z+\gamma^{2} p(2 p-B p+1)<(z-\gamma p) \sqrt{\Delta}
$$


We know that both sides are equal for $z=\gamma \frac{1-p \pi_{h}}{1-\pi_{h}}$, and that the left-hand side is a negative and right left-hand side is positive for $z$ high enough, so either $\frac{\gamma\left(1-\pi_{r} p\right)}{\left(1-\pi_{r}\right)}<z$ is satisfied for $z \in\left(z_{h}, z_{h}+\varepsilon\right)$, or not, in which case there exists $z^{\prime}>z_{h}$ for which $\frac{\gamma\left(1-\pi_{r}\left(z^{\prime}\right) p\right)}{\left(1-\pi_{r}\left(z^{\prime}\right)\right)}=z^{\prime}$ and $\frac{\gamma\left(1-\pi_{r}(z) p\right)}{\left(1-\pi_{r}(z)\right)}<z$ for all $z>z^{\prime}$

Combining Proposition 8 with 9, we see that there exist parameters for which increasing misconduct discoverability $p$ decreases misbehavior and at the same time increases the production of scandals. This finding provides further reason to exercise caution when inferring misconduct from scandal. Indeed, institutions and jurisdictions with lower rates of misbehavior may experience more scandals than those with higher rates.

The findings on the political consequences of scandal presented in Proposition 6 carry through when misbehavior is endogenized. Moreover, Proposition 8 implies that we are likely to be in the $\pi^{*} p<\frac{1}{2}$ regime when stakes of an election $x(1-c)$ are small, and in $\pi^{*} p>\frac{1}{2}$ otherwise. Hence, when stakes of election are large for the parties, scandals have little impact on the politician but do substantial reputational damage to the aligned party. ${ }^{25}$

Lastly, identifying conditions under which voters make inferential errors is even more complex than previously recognized. For example, Proposition 2 says that as stakes of an election $x(1-c)$ increase, voters are more likely to make a wrong decision about a politician's fate. But since an increase in $x(1-c)$ increases the equilibrium incidence of misbehavior $\pi^{*}$, a second effect arises, in which the probability of making the wrong decision first increases and then decreases in the equilibrium incidence of misbehavior $\pi^{*}$. Hence, if we start in an environment with low levels of misbehavior, then as in the exogenous misbehavior case, increases in the stakes of an election $x(1-c)$ compromise the voter's ability to correctly infer misbehavior. We cannot rule out that the reverse holds, however, if we start in an environment with high levels of misbehavior.

\footnotetext{
${ }^{25}$ Since $\pi^{*}$ is decreasing in $p$, the set of $p$ for which $\pi^{*} p<\frac{1}{2}$ is likely to be more complex, and we refrain from characterizing such a set recognizing that our results would depend upon the distributional assumptions we make about $b$. The distributional assumptions are unlikely to affect the comparative statics results that we present in this section. However, they could affect the set of $p$ for which we have $\pi^{*} p<\frac{1}{2}$. If benefits from misconduct are likely to be large, for instance, then we can expect $\pi^{*}$ to be large for many values of $p$. If not, $\pi^{*} p<\frac{1}{2}$ may obtain for all values of $p$.
} 


\subsection{Characterization of the Remaining Equilibria}

To simplify the exposition, for this part we set $p=1$, but the logic of the proof does not depend on this choice. In the interest of space, denote the parties by $a$ for aligned and $o$ for opposition. Let $s_{i}$ and $f_{i}$ be the probability that party $i \in\{a, o\}$ suppresses and fabricates, respectively. In our model, parties' behavior is unconstrained by what information they have, but the information they have tells them something about the behavior of the other party. In particular, upon seeing misconduct, each party knows that the other party reveals this information if it happens to be honest. Hence, the incentives of each party to choose $a_{i} \in\{0,1\}$ depend on its information.

Proposition 10 If $\gamma<\pi<1-\gamma$, then there exists an equilibrium in which

$$
s_{a}=s_{o}=\frac{1-\pi}{1-\gamma} \text { and } f_{o}=f_{a}=\frac{\pi}{1-\gamma}
$$

In this equilibrium, each party is indifferent between $a_{i}=1$ and $a_{i}=0$ independent of the information they posses, and the voter does not update about the parties and the politician. There exists no other equilibrium with $s_{i} \in(0,1)$ and $f_{i} \in(0,1)$ for some $i \in\{a, o\}$.

Proof. Bayes rule implies that as long as well defined, the beliefs are as follows

$$
\begin{gathered}
\phi_{a}(1,1)=\frac{\pi\left(\gamma+(1-\gamma)\left(1-s_{o}\right)\right) \gamma}{\pi\left(\gamma+(1-\gamma)\left(1-s_{o}\right)\right) \gamma+\left(\pi\left(1-s_{a}\right)\left(\gamma+(1-\gamma)\left(1-s_{o}\right)\right)+(1-\pi) f_{a}(1-\gamma) f_{o}\right)(1-\gamma)}, \\
\phi_{a}(0,1)=\frac{(1-\pi)(1-\gamma) f_{o} \gamma}{(1-\pi)(1-\gamma) f_{o} \gamma+\left(\pi s_{a}\left(\gamma+(1-\gamma)\left(1-s_{o}\right)\right)+(1-\pi)\left(1-f_{a}\right)(1-\gamma) f_{o}\right)(1-\gamma)}, \\
\phi_{a}(1,0)=\frac{\pi(1-\gamma) s_{o} \gamma}{\pi(1-\gamma) s_{o} \gamma+\left(\pi\left(1-s_{a}\right)(1-\gamma) s_{o}+(1-\pi) f_{a}\left(\gamma+(1-\gamma)\left(1-f_{o}\right)\right)\right)(1-\gamma)}, \\
\phi_{a}(0,0)=\frac{(1-\pi)\left(\gamma+(1-\gamma)\left(1-f_{o}\right)\right) \gamma}{(1-\pi)\left(\gamma+(1-\gamma)\left(1-f_{o}\right)\right) \gamma+\left(\pi s_{a}(1-\gamma) s_{o}+(1-\pi)\left(1-f_{a}\right)\left(\gamma+(1-\gamma)\left(1-f_{o}\right)\right)\right)(1-\gamma)}, \\
\phi_{o}(1,1)=\frac{\pi\left(\gamma+(1-\gamma)\left(1-s_{a}\right)\right) \gamma}{\pi\left(\gamma+(1-\gamma)\left(1-s_{a}\right)\right) \gamma+\left(\pi\left(1-s_{o}\right)\left(\gamma+(1-\gamma)\left(1-s_{a}\right)\right)+(1-\pi) f_{o}(1-\gamma) f_{a}\right)(1-\gamma)}, \\
\phi_{o}(1,0)=\frac{(1-\pi)(1-\gamma) f_{a} \gamma}{(1-\pi)(1-\gamma) f_{a} \gamma+(1-\gamma)\left(\pi s_{o}\left(\gamma+(1-\gamma)\left(1-s_{a}\right)\right)+(1-\pi)\left(1-f_{o}\right)(1-\gamma) f_{a}\right)}, \\
\phi_{o}(0,0)=\frac{(1-\pi)\left(\gamma+(1-\gamma)\left(1-f_{a}\right)\right) \gamma}{(1-\pi)\left(\gamma+(1-\gamma)\left(1-f_{a}\right)\right) \gamma+(1-\gamma)\left(\pi s_{o}(1-\gamma) s_{a}+(1-\pi)\left(1-f_{o}\right)\left(\gamma+(1-\gamma)\left(1-f_{a}\right)\right)\right)} \\
\phi_{o}(0,1)=\frac{\pi(1-\gamma) s_{a} \gamma}{\pi(1-\gamma) s_{a} \gamma+\left(\pi\left(1-s_{o}\right)(1-\gamma) s_{a}+(1-\pi) f_{o}\left(\gamma+(1-\gamma)\left(1-f_{a}\right)\right)\right)(1-\gamma)},
\end{gathered}
$$




$$
\begin{aligned}
& \Phi(1,0)=\frac{\left(\gamma+(1-\gamma)\left(1-s_{a}\right)\right)(1-\gamma) s_{o} \pi}{\left(\gamma+(1-\gamma)\left(1-s_{a}\right)\right)(1-\gamma) s_{o} \pi+(1-\gamma) f_{a}\left(\gamma+(1-\gamma)\left(1-f_{o}\right)\right)(1-\pi)} \\
& \Phi(1,1)=\frac{\left(\gamma+(1-\gamma)\left(1-s_{a}\right)\right)\left(\gamma+(1-\gamma)\left(1-s_{o}\right)\right) \pi}{\left(\gamma+(1-\gamma)\left(1-s_{a}\right)\right)\left(\gamma+(1-\gamma)\left(1-s_{o}\right)\right) \pi+(1-\gamma) f_{a}(1-\gamma) f_{o}(1-\pi)} \\
& \Phi(0,1)=\frac{(1-\gamma) s_{a}\left(\gamma+(1-\gamma)\left(1-s_{o}\right)\right) \pi}{(1-\gamma) s_{a}\left(\gamma+(1-\gamma)\left(1-s_{o}\right)\right) \pi+\left(\gamma+(1-\gamma)\left(1-f_{a}\right)\right)(1-\gamma) f_{o}(1-\pi)} \\
& \Phi(0,0)=\frac{(1-\gamma) s_{a}(1-\gamma) s_{o} \pi}{(1-\gamma) s_{a}(1-\gamma) s_{o} \pi+\left(\gamma+(1-\gamma)\left(1-f_{a}\right)\right)\left(\gamma+(1-\gamma)\left(1-f_{o}\right)\right)(1-\pi)}
\end{aligned}
$$

Suppose $m=v=1$. Then the aligned party prefers not to suppress if and only if

$$
\begin{aligned}
& \left(\gamma+(1-\gamma)\left(1-s_{o}\right)\right)\left(\phi_{a}(1,1)-\phi_{a}(0,1)+(\Phi(0,1)-\Phi(1,1)) z\right) \\
& \quad+(1-\gamma) s_{o}\left(\phi_{a}(1,0)-\phi_{a}(0,0)+(\Phi(0,0)-\Phi(1,0)) x\right) \geq 0
\end{aligned}
$$

Suppose $m=v=0$. Then the aligned party weakly prefers to fabricate if and only if

$$
\begin{gathered}
(1-\gamma) f_{o}\left(\phi_{a}(1,1)-\phi_{a}(0,1)+(\Phi(0,1)-\Phi(1,1)) z\right) \\
+\left(\gamma+(1-\gamma)\left(1-f_{o}\right)\right)\left(\phi_{a}(1,0)-\phi_{a}(0,0)+(\Phi(0,0)-\Phi(1,0)) z\right) \geq 0
\end{gathered}
$$

Denoting

$$
\begin{aligned}
A & \equiv \phi_{a}(1,1)-\phi_{a}(0,1)+(\Phi(0,1)-\Phi(1,1)) z, \\
B & \equiv \phi_{a}(1,0)-\phi_{a}(0,0)+(\Phi(0,0)-\Phi(1,0)) z,
\end{aligned}
$$

we can write

$$
[a \text { prefers not to suppress }] \Leftrightarrow\left(\gamma+(1-\gamma)\left(1-s_{o}\right)\right) A+(1-\gamma) s_{o} B \geq 0
$$

$$
[a \text { prefers to fabricate }] \Leftrightarrow(1-\gamma) f_{o} A+\left(\gamma+(1-\gamma)\left(1-f_{o}\right)\right) B \geq 0 \text {. }
$$


Similarly, using notation

$$
\begin{aligned}
C & \equiv \phi_{o}(1,1)-\phi_{o}(1,0)-(\Phi(1,0)-\Phi(1,1)) z \\
D & \equiv \phi_{o}(0,1)-\phi_{o}(0,0)-(\Phi(0,0)-\Phi(0,1)) z
\end{aligned}
$$

we have that

$$
\begin{gathered}
{[o \text { prefers not to suppress }] \Leftrightarrow\left(\gamma+(1-\gamma)\left(1-s_{a}\right)\right) C+(1-\gamma) s_{a} D \geq 0,} \\
{[o \text { prefers to fabricate }] \Leftrightarrow(1-\gamma) f_{a} C+\left(\gamma+(1-\gamma)\left(1-f_{a}\right)\right) D \geq 0 .}
\end{gathered}
$$

The following lemma states that if one party behaves honestly in one information state, then the other party has to behave honestly in some information state.

Lemma 2 If $s_{i}=0$, then either $f_{-i}=0$ or $s_{-i}=0$. If $f_{i}=0$, then either $f_{-i}=0$ or $s_{-i}=0$.

Proof. Consider $s_{o}=0$, then one can easily show that $B<0$. If $A \leq 0$, then from from (31), a strictly prefers not to fabricate and hence $f_{a}=0$, and if $A>0$, and then from from (30), a strictly prefers not to suppress and hence $s_{a}=0$. Consider $f_{o}=0$, then one can easily show that $A>0$. If $B \geq 0$, then from from (30), a prefers not to suppress and hence $s_{a}=0$, and if $B<0$, then from from (31), a prefers not to fabricate, and hence $f_{a}=0$.

The lemma above implies that in equilibrium, either $f_{i}>0$ and $s_{i}>0$ for both $i \in\{a, o\}$, or $\left(s_{o}=s_{a}=0\right)$, or $\left(s_{o}=f_{a}=0\right)$, or $\left(f_{o}=s_{a}=0\right)$, or $\left(f_{o}=f_{a}=0\right)$. In the main text, we characterize all equilibria in which $\left(s_{o}=f_{a}=0\right)$. From the equilibrium conditions, for $s_{o}=s_{a}=0$ to be an equilibrium, (30) requires $A \geq 0$, but plugging $s_{o}=s_{a}=0$ into $A$, we obtain that $A<0$, which is a contradiction. From the equilibrium conditions, for $f_{o}=f_{a}=0$ to be an equilibrium, (31) requires $B \leq 0$ and $D \leq 0$, but plugging $f_{o}=f_{a}=0$ into $D$, we obtain that $D \geq 0$, which is a contradiction. And finally, using $\left(f_{o}=s_{a}=0\right)$ in $B$ and $C$, we obtain that $B<0$ and $C>0$, which together with (31) and (32) implies that $f_{a}=0$ and $s_{o}=0$.

So in any equilibrium not analyzed in the paper it must be that $f_{i}>0$ and $s_{i}>0$ for both $i \in\{a, o\}$, which means that (30), (31), (32), and (33) are satisfied. Consider first an 
equilibrium in which $f_{a} \in(0,1)$ and $s_{a} \in(0,1)$, that is, the aligned party fully randomizes. That requires that (30) and (31) are satisfied with equality, which this requires that either $A=B=0$, or $f_{o}=\frac{1}{(1-\gamma)}-s_{o}$. We consider first $f_{o}=\frac{1}{(1-\gamma)}-s_{o}$, and treat the case of $A=B=0$ with $f_{o} \neq \frac{1}{(1-\gamma)}-s_{o}$ in the next paragraph. Plugging $f_{o}=\frac{1}{(1-\gamma)}-s_{o}$ into $A$ and $B$, one sees that $A=B$, so we still need $A=B=0$, which requires $f_{a}=\frac{\pi}{1-\gamma}$ and $s_{a}=\frac{1-\pi}{1-\gamma}$. Plugging $\left(f_{a}=\frac{\pi}{1-\gamma}, s_{a}=\frac{1-\pi}{1-\gamma}\right)$ into $C$ and $D$, one can see that $C=D$. This implies that either $C=D=0$ and both (32) and (33) are satisfied with equality, or $C=D \neq 0$, in which case $f_{o}=1-s_{o}=1$ or $f_{o}=1-s_{o}=0$. But the latter contradicts the supposition that $f_{o}=\frac{1}{(1-\gamma)}-s_{o}$, so (32) and (33) are satisfied with equality, which delivers that $s_{o}=\frac{1-\pi}{1-\gamma}$ and $f_{o}=\frac{\pi}{1-\gamma}$. And $f_{i} \in(0,1)$ and $s_{i} \in(0,1)$ requires that $\gamma<\pi<1-\gamma$.

Consider still an equilibrium in which $f_{a} \in(0,1)$ and $s_{a} \in(0,1)$, but suppose that $f_{o} \neq \frac{1}{(1-\gamma)}-s_{o}$, so (30), (31) are satisfied with equality because $A=B=0$. Using the formulas for $A$ and $B$, and isolating $z$ from $A=0, B=0$, we obtain

$$
\begin{aligned}
& z=\gamma\left(\pi+f_{o}(1-\gamma)(1-\pi)-\pi s_{o}(1-\gamma)\right) \frac{-\pi s_{a}+f_{a} f_{o}(1-\gamma)(1-\pi)+\pi s_{a} s_{o}(1-\gamma)}{\pi f_{o}\left(-s_{o}+\gamma s_{o}+1\right)\left(-f_{a}-s_{a}+\gamma f_{a}+\gamma s_{a}+1\right)(\pi-1)}, \\
& z=\gamma\left(\pi+f_{o}(1-\gamma)(1-\pi)-\pi s_{o}(1-\gamma)-1\right) \frac{-f_{a}+\pi f_{a}+f_{a} f_{o}(1-\gamma)(1-\pi)+\pi s_{a} s(1-\gamma)}{\pi s_{o}\left(-f_{o}+\gamma f_{o}+1\right)\left(-f_{a}-s_{a}+\gamma f_{a}+\gamma s_{a}+1\right)(\pi-1)} .
\end{aligned}
$$

The right-hand sides of these formulas are identical if $f_{a} f_{o}(\pi-1)^{2}\left(-f_{o}+\gamma f_{o}+1\right)$ $+\pi^{2} s_{a} s_{o}\left(-s_{o}+\gamma s_{o}+1\right)=0$, which is impossible. So there is no other equilibrium with $f_{a} \in(0,1)$ and $s_{a} \in(0,1)$.

An analogous argument developed for the opposition party delivers that the equilibrium described in (27) is the only equilibrium with $f_{o} \in(0,1)$ and $s_{o} \in(0,1)$. So the only other possibilities are equilibria in which $\left(s_{a}=s_{o}=1\right)$, or $\left(s_{a}=f_{o}=1\right)$, or $\left(f_{a}=s_{o}=1\right)$ or $\left(f_{a}=f_{o}=1\right)$. From those, it is very easy to rule out $\left(f_{a}=s_{a}=f_{a}=f_{o}=1\right)$. 


\section{References}

Adut, Ari. 2008. On Scandal: Moral Disturbances in Society, Politics and Art. New York, NY: Cambridge University Press.

Adut, Ari. 2005. "A Theory of Scandal: Victorians, Homosexuality, and the Fall of Oscar Wilde. American Journal of Sociology 111 (July): 213-248.

Anderson, Christopher and Yuliya Tverdova. 2003. "Corruption, Political Allegiances, and Attitudes Toward Government in Contemporary Democracies." American Journal of Political Science 47(1): 91-109.

Andolina, Molly and Clyde Wilcox. 2000. "Public Opinion: The Paradoxes of Clinton's Popularity." In Mark Rozell and Clyde Wilcox (eds), The Clinton Scandals and the Future of American Government. Washington, D.C.: Georgetown University Press.

Banerjee, Abhijit, Donald P. Green, Jeffery McManus, and Rohini Pande. 2014. "Are Poor

Voters Indifferent to whether Elected Leaders are Criminal or Corrupt? A Vignette Experiment in Rural India. Political Communication 31(3): 391-407.

Basinger, Scott and Brandon Rottinghaus. 2012. "Stonewalling: Explaining Presidential Behavior during Scandal." Political Research Quarterly 65(2): 290-302.

Besley, Tim and Prat, Andrea. 2006. "Handcuffs for the Grabbing Hand?: Media Capture and Government Accountability." American Economic Review 96 (3): 720-736.

Bongino, Dan and D.C. McAllister. 2018. 'Spygate: The Attempted Sabotage of Donald J. Trump New York, NY: Post Hill Press.

Bowler, Shaun and Jeffrey Karp. 2004. "Politicians, Scandals and Trust in Government." Political Behavior. 26(3): 271-87.

Boylan, Richard and Cheryl Long. 2003. "Measuring Public Corruption in the American States: A Survey of state House Reporters." State Politics and Policy Quarterly 3(4): 420-38.

Cameron, Charles. 2002. "Studying the Polarized Presidency." Presidential Studies Quarterly. 32(4): 647-663.

Carlson, James, Gladys Ganiel, and Mark S. Hyde. 2000. "Scandal and Political Candidate Image. Southeastern Political Review 28(4): 747-757. 
Chaffee, S.H. and L.B. Becker. 1975. "Young Voters' Reactions to Early Watergate Issues." American Politics Quarterly. 3(4): 360-385.

Chassang, S. and G. Padro i Miquel. Forthcoming. "Crime, Intimidation, and Whistleblowing: A Theory of Inference from Unverifiable Reports." Review of Economic Studies

Chong, Alberto, Ana De La O, Dean Karlan, and Leonard Wantchekon. 2015. "Does Corruption Information Inspire the Fight or Quash the Hope? A Field Experiment in Mexico on Voter Turnout, Choice, and Party Identification. The Journal of Politics 77(1): $55-71$.

Coen, Jeff and John Chase. 2012. Golden: How Rod Blagojevich Talked Himself out of the Governor's Office and into Prison. Chicago, IL: Chicago Review Press.

Corinta, Jeronimo and Brandon Rottinghaus. 2017 "Does Partisanship Stop at Scandal's Edge? Partisan Resiliency and the Survival of Political Scandal." American Review of Politics 36(1): 63-77.

Dewan, Torun and David Myatt. 2007. "Scandal: Protection and Recovery in the Cabinet." American Political Science Review 101(1): 63-77.

Dewberry, David. 2015. The American Political Scandal: Free Speech, Public Discourse, and Democracy. New York, NY: Roman and Littlefield.

Dunlap, Riley E. and Robert L. Wisniewski 1978. "The Effect of Watergate on Political Party Identification: Results from a 1970-74 Panel Study." Sociological Focus 11(2): 69-80.

Eggers, Andrew C. 2014. "Partisanship and Electoral Accountability: Evidence from the UK Expenses Scandal", Quarterly Journal of Political Science 9(4): 441-472.

Entman, Robert. 2012. Scandal and Silence: Media Responses to Presidential Misconduct. Malden, MA: Polity.

Ferraz, Claudio and Frederico Finan. 2011. "Electoral Accountability and Corruption: Evidence from the Audits of Local Governments." American Economic Review 101(4): 1274-14.

Garment, Suzanne. 1991. Scandal: The Culture of Mistrust in American Politics. New York, NY: Anchor Books.

Gentzkow, Matthew, Edward L. Glaeser, and Claudia Goldin. 2004. "The Rise of the 
Fourth Estate: How Newspapers Became Informative and Why it Mattered." in Edward L. Glaeser and Claudia Goldin, eds., Corruption and Reform: Lessons from America's Economic History. University of Chicago Press.

Gratton, Gabriele, Richard Holden and Anton Kolotilin. 2018. "When to Drop a Bombshell." Review of Economics Studies 85(4): 2139-2172.

Green, Donald, Adam Zelizer and David Kirby. 2018. "Publicizing Scandal: Results from Five Field Experiments." Quarterly Journal of Political Science. 13(3): 237-61.

Harding, Luke. 2017. Collusion: Secret Meetings, Dirty Money, and How Russia Helped Donald Trump Win. New York, NY: Vintage.

Hirano, Shigeo and James Snyder. 2018. Primary Elections in the United States. Mimeo.

Invernizzi, Giovanna. 2016. "Political Scandals." Columbia University mimeo.

Jacobson, Gary and M. Dimock 1994. "Checking Out: The Effects of Bank Overdrafts on the 1992 House Elections." American Journal of Political Science. 601-624.

Klasnja, Marko. 2017. "Uninformed Voters and Corrupt Politicians. American Politics Research 45(2): 256-279.

Lipset, Seymour Martin and W. Schneider. 1983. "The Decline of Confidence in American Institutions." Political Science Quarterly. 98(3): 379-402.

Lowi, Theodore. 2018. Forward to The Politics of Scandal: Power and Process in Liberal Democracies Primary Elections in the United States. Edited by Andrei Marcovits and Mark Silverstein. New York, NY: Holmes and Meier.

Meier, Kenneth and Thomas Holbrook. 1992. "I seen My Opportunities and I took them: Political Corruption in the American States." Journal of Politics. 54(2): 135-55.

Meinke, Scott and William Anderson. 2001. "Influencing from Impaired Administrations: Presidents, White House Scandals and Legislative Leadership." Legislative Studies Quarterly 26(4): 639-59.

Miller, Arthur. 1999. "Sex, Politics, and Public Opinion: What Political Scientists Really Learned from the Clinton-Lewinsky Scandal." PS: Political Science 32(4): 721-29.

Mishler, William and Richard Rose. 2001. "What Are the Origins of Political Trust? Testing Institutional and Cultural Theories in Post-Communist Societies." Comparative Political Studies 34(1): 30-62. 
Nyhan, Brendan. 2015. "Scandal Potential: How Political Context and News Congestion Affect the President's Vulnerability to Media Scandal. British Journal of Political Science 45 (2): 435-466.

Nyhan, Brendan. 2017. "Media Scandals Are Political Events: How Contextual Factors Affect Public Controversies over Alleged Misconduct by U.S. Governors." Political Research Quarterly 70(1): 223-236.

Nyhan, Brendan and Jason Reifler. 2015. Displacing Misinformation about Events: An Experimental Test of Causal Corrections. Journal of Experimental Political Science 2(1): 8193.

Pereira, Miguel and Nichols Waterbury. 2018. "Do Voters Discount Political Scandals over Time." Political Research Quarterly.

Persson, Torsten, Guido Tabellini and Francesco Trebbi. 2003. "Electoral rules and corruption." Journal of the European Economic Association 1(4): 958-989.

Puglisi, Riccardo and James Snyder. 2011. "Newspaper Coverage of Political Scandals." Journal of Politics. 73(3): 931-50.

Renshon, Stanley. 2002. "The Polls: The Public's Response to the Clinton Scandals. Part 1: Inconsistent Theories, Contradictory Evidence." Presidential Studies Quarterly. 32(1): 169-184.

Robinson, John P. 1974. "Public Opinion during the Watergate Crisis." Communication Research. 1(4): 391-405.

Rottinghaus, Brandon. 2015. The Institutional Effects of Executive Scandal. New York, NY: Cambridge University Press.

Sabato, Larry, Mark Stencel, and S. Robert Lichter. 2001. Peepshow: Media and Politics in the Age of Scandal. Lanham, MD: Rowman \& Littlefield.

Simon, Dennis and Charles Ostrom. 1989. "The Impact of Televised Speeches and Foreign Travel on Presidential Approval." Public Opinion Quarterly 53(1): 53-82.

Thompson, John. 2000. Political Scandal: Power and Visibility in the Media Age. New York, NY: Cambridge University Press.

Toobin, Jeffrey. 2000. A Vast Conspiracy: The Real Story of the Sex Scandal that Nearly Brought Down a President. New York, NY: Touchstone. 
Welch, Susan and John Hibbing. 1997. "The Effect of Charges of Corruption on Voting Behavior in Congressional Elections, 1982-1990." Journal of Politics. 59(1): 226-39.

Whyte, Kenneth. 2017. Hoover: An Extraordinary Life in Extraordinary Times. New York, NY: Alfred A. Knopf.

Woessner, Matthew. 2005. "Scandal, Elites and Presidential Popularity: Considering the Importance of Cues in Public Support of the President." Presidential Studies Quarterly. 35: $94-115$.

Woodward, Bob and Carl Bernstein. 1974. All the President's Men. New York, NY: Simon $\&$ Schuster.

Woodward, C. Vann 1974. The Conscience of the White House in Presidential Misconduct: From George Washington to Today. Ed James M Banner, The New Press.

Zaller, John. 1998. "Monica Lewinsky's Contribution to Political Science." PS: Political Science 31(2): 182-89. 